\title{
Studies of nano-structured liquids in confined geometries and at surfaces.
}

\author{
J. Beau W. Webber*,a,b \\ ${ }^{a}$ Institute of Petroleum Engineering, Heriot-Watt University, Edinburgh. EH14 $4 \mathrm{AS}$ \\ ${ }^{b}$ School of Physical Sciences, University of Kent, Canterbury, Kent. CT2 7NH
}

Key words:

liquids, water, ice, confined geometry, pores, NMR relaxation, neutron scattering, cryoporometry

PACS: 64.60.-i, 65.60.+a, 81.07.-b, 03.75.Hh, 05.70.Fh, 64.60.Qb, 64.70.Dv, 68.03.Cd, 68.08.-p, 82.56.Na, 82.56.Ub, 61.43.Fs, 61.43.Gt, 61.46.+w, 82.60.Qr

\section{Contents}

1 Introduction. $\quad 3$

1.1 Samples. . . . . . . . . . . . . . . 5

1.2 Methods. . . . . . . . . . . . . . . . . . . 6

2 The thermodynamics of liquids in nano-pores. 6

2.1 The geometry term in the Gibbs-Thomson equation. . . . . . 9

2.2 Second order (linearity) terms in the Gibbs-Thomson equation. 10

${ }^{*}$ Correspondence address: Beau Webber, Lab-Tools Ltd., Canterbury Enterprise Hub, University of Kent, Canterbury, Kent. CT2 7NJ. +44 (0) 1227824675

Email address: J.B.W.Webber@kent.ac.uk (J. Beau W. Webber)

$U R L:$ http://www.Lab-Tools.com (J. Beau W. Webber) 
3 The application of NMR relaxation, diffusion and cryoporometry techniques to the study of the properties of liquids in pores.

4 NMR Cryoporometry : experimental.

4.1 NMR Cryoporometry : experimental calibration of $k_{G T}$ for the melting transition. . . . . . . . . . . . . . 16

4.2 NMR Cryoporometry : experimental effect of measuring time on the measured melting transition. . . . . . . . . . . . 20

4.3 NMR Cryoporometry : experimental calibration of $k_{G T}$ for the freezing transition. . . . . . . . . . . . . . 21

4.4 Effects of interface geometries on freezing/melting behaviour : experimental. . . . . . . . . . . . 26

5 Water/ice at an interface : Plastic Ice. $\quad 27$

5.1 Water/ice at a silica interface . . . . . . . . . . . 27

5.1.1 Structure, as determined by Neutron diffraction cryoporometry, using a global analysis, with a fully-filled sample . . . . . . . . . . . . . . . 27

5.1.2 Dynamics, as determined by NMR transverse relaxation, for plastic ice in a fully-filled sample. . . . . . . 31

5.2 Water/ice at a vapour interface . . . . . . . . . . . . . 39

5.2.1 Dynamics, as determined by NMR transverse relaxation, for plastic ice in a partially-filled sample. . . . . 39 
5.2.2 Structure, as determined by Neutron diffraction cryoporometry, using a global analysis, with a partiallyfilled sample. . . . . . . . . . . . . . . . 42

5.2.3 Structure, as determined be Neutron diffraction, using a peak analysis, with a partially-filled sample. . . . . . 44

5.2.4 Structure, as determined by Neutron diffraction, using a radial domain analysis. . . . . . . . . . . . . . . 47

6 Applications and Consequences.

6.1 Relevance to NMR cryoporometry in short $T_{2}$ materials : meteorite example. . . . . . . . . . . . . . 47

6.2 Relevance to macroscopic snow-packs in the environment. . . . 51

7 Acknowledgments

\section{Introduction.}

This is a progress report on elucidating the behaviour of liquids, in particular water, in confined geometry on the nano- to meso-scale, and at interfaces. There are important measurements still to make, conclusions still to be drawn, and above all leaps of understanding still to be made. However a number of important features in the behaviour of these systems have recently become clearer.

Nano-structuring of liquids and their crystals changes their Gibbs Free Energy, and hence their dynamics. This may most readily be probed by monitoring the alteration of phase changes as a function of temperature, together with changes in other parameters, particularly the confinement diameter. 
Such studies may be performed by monitoring the change in the pressure (at constant temperature) of the liquid in its own vapour (Kelvin Equation), or by monitoring the change in the freezing/melting temperature (at constant pressure) of a crystal in its own liquid (Gibbs-Thomson Equation).

In the latter case the melting and freezing temperatures of liquids are modified by the changes in the volumetric Gibbs Free Energy due to nanostructuring; this is related to the surface energy of the curved interface between the crystal and its own liquid. This is thus dependent on the geometry of the interface between the crystal and its liquid. There is still discussion on this point as to the exact geometric constants and functional forms that are applicable for different confining geometries. Experimental evidence is presented for the cases of cylindrical pores (SBA-15), and for pores that on average are spherical (sol-gel). However reconciling this comparative data with melting/freezing temperatures in each of these systems still poses a number of questions.

It is well known that bulk brittle ice has a hexagonal stucture, while brittle ice that forms in pores may be cubic in structure [1, 2], figures 10, 11. Adjacent surfaces appear to further alter the dynamics and structure of confined liquids and their crystals, leading in the case of a water/ice system to a state of enhanced rotational motion (plastic ice) just below the confined freezing/melting transitions. This plastic ice layer appears to form at both the ice-silica interface and the ice-vapour surface, and reversibly transforms to brittle ice at lower temperatures. There is good evidence to suggest that the plastic ice at a silica interface transforms to cubic ice, while the plastic ice at vapour surfaces transforms to hexagonal ice. That this plastic ice 
may correspond to a layer at the crystal surface is suggested by the presence of only amorphous ice in confined systems with small dimensions $(<\sim 3 \mathrm{~nm}$ diameter), whereas systems with larger dimensions $(\sim 10 \mathrm{~nm})$ contain brittle cubic ice and also some hexagonal ice (if a vapour interface is present); even larger systems $(>\sim 30 \mathrm{~nm})$ contain predominately hexagonal ice. It is conjectured that this layer of plastic ice at vapour surfaces may be present at the myriad of such interfaces in macroscopic systems, such as snow-packs, glaciers and icebergs, and may be an explanation for the need for plastic terms in the macroscopic dynamical models of these systems [3].

These results also point the way forward for a wide-range of cryoporometric metrology studies of systems that are 'difficult' for NMR, such as high iron content clays and rocks, as well as aged concrete. Results are presented for cryoporometric measurements on meteorite samples with a significant metallic content, exhibiting $T_{2}^{*}$ relaxation times down to $2.5 \mu \mathrm{s}$.

\subsection{Samples.}

The nano-structuring of liquids greatly modifies their physical properties. Confining liquids in materials with pores, in order to study these properties, has a long history. Because of the large surface area within these pores, placing liquids in pores also forms an excellent method of studying the properties of liquids near surfaces (which have been shown to behave differently from the rest of the liquid in the pore). There are many informative studies of liquids in zeolites, sol-gel silicas and in the regular templated silicas such as MCM-41, MCM-48. However the newer templated silicas, such as SBA-15 and SPS-1, have expanded the range of sizes available from regular templated silicas, and studies in these have been particularly informative, their narrow 
pore size distributions enabling a precise probing of liquid properties as a function of distance from the pore surface.

\subsection{Methods.}

One method of studying these systems, that has been shown to be of great importance, involves measuring the phase-change properties of liquids in nano- to meso-pores over a wide temperature range : this reveals a wide range of phase change behaviour in such liquids compared with that in the bulk liquid. Most importantly these phase changes give us useful probes into the physics that is controlling the dynamics in the confined geometry. NMR relaxation studies provide a further method of probing the dynamics, as does quasi-elastic neutron scattering (QENS), while neutron diffraction, small angle neutron scattering (SANS) and neutron diffraction cryoporometry (NMR-C) are also powerful tools for probing the structure.

\section{The thermodynamics of liquids in nano-pores.}

J.W. Gibbs, J. Thomson, W. Thomson (later Lord Kelvin) and J.J. Thomson employed thermodynamics, generalised dynamics and experimentation to develop a set of equations describing the effects that a range of variables, including geometry, have on basic properties of matter such as vapour pressure and melting point $[4,5,6,7,8,9]$. In particular there are important behaviours that are closely related to the capillary effect, that reflect the change in bulk free energy caused by the curvature of an interfacial surface under tension $[10,11]$.

For these systems the constant temperature variant of the Gibbs equations is the Kelvin Equation, which describes the change in vapour pressure 
with radius of curvature, for a small isolated droplet of liquid in its own vapour :

$$
R T \ln \frac{P_{v}}{P_{0}}=-2 \gamma \frac{V_{M}}{R_{k}}
$$

where $R$ is the gas constant, $T$ the absolute temperature, $P_{0}$ the ambient pressure, $P_{v}$ the vapour pressure $\gamma$ is the liquid surface tension at temperature $T, V_{M}$ is the molar volume of the liquid and $R_{k}$ is the Kelvin radius.

Equation 1 can also be applied to the model of a right cylindrical void containing a hemispherical interface between a wetting liquid and its own vapour [9], where the presence of a confining geometry requires an additional $\cos (\phi)$ term to be incorporated into the Kelvin equation to accommodate the interaction between the absorbate and the pore walls :

$$
R T \ln \frac{P_{v}}{P_{0}}=-2 \gamma \frac{V_{M}}{R_{k}} \cos \phi
$$

where $\phi$ is the contact angle between the liquid and vapour at the pore wall.

Equation 2 describes the evaporation branch of the absorption isotherms $[12,13,11]$, since condensation is believed to occur initially as monolayers (i.e. with cylindrical geometry in cylindrical pores), but evaporation is believed to occur at a hemispherical interface that travels along the pore as it empties.

The constant pressure variant of the Gibbs equations is the Gibbs-Thomson Equation, which describes the change in melting temperature with radius of curvature for a small crystal. Consequently, the Gibbs-Thomson equation for the melting point depression, $\Delta T_{m}$, for a small isolated crystal of diameter $x$ in its own liquid may be approximated [14] as :

$$
\Delta T_{m}=T_{m}^{\infty}-T_{m}(x)=\frac{4 \sigma_{c l} T_{m}^{\infty}}{x \Delta H_{f} \rho_{s}}
$$


where : $T_{m}^{\infty}=$ normal melting point of bulk liquid (considered to be a crystal of infinite size), $T_{m}(x)=$ melting point of crystals of diameter $x, \sigma_{c l}=$ surface energy at the crystalline-liquid interface, $\Delta H_{f}=$ bulk enthalpy of fusion (per gram of material), $\rho_{s}=$ density of the solid.

Equation 3 can also be applied to the model of a right cylindrical void containing a hemispherical interface between a crystal and its own liquid, and again the presence of a confining geometry requires an additional $\cos (\phi)$ :

$$
\Delta T_{m}=T_{m}^{\infty}-T_{m}(x)=\frac{4 \sigma_{c l} T_{m}^{\infty}}{x \Delta H_{f} \rho_{s}} \cos \phi
$$

The contact angle $\phi$ is commonly assumed to be $0^{\circ}$ in the Kelvin case and $180^{\circ}$ in the Gibbs-Thomson case [15], [16, VII - Cohesive Attraction of Solids and Fluids pp 432-436, partially ascribed to Clairaut], [11, Eqns. 3.1, 3.2, 3.27, pp 123, 135-136, 150-156], [17, Eqn. 2], [14, p 9002], [18]. the dependance of $\Delta T_{m}$ on $\cos \phi$ has a straightforward geometric interpretation since a spherical meniscus of radius $r_{1}$, in a cylindrical capillary of radius $r$, with an angle of contact $\phi$, has $r=r_{1} \cos \phi[11]$.

If, with suffixes l: liquid, s: solid, p: pore wall, we apply the Young equation $\sigma_{p l}=\sigma_{s l} \cos (\phi)+\sigma_{p s}$ and the Young-Dupré equation $W_{p s}=\sigma_{s l}+$ $\sigma_{p l}-\sigma_{p s}$, being the reversible work required to separate unit area of the solid from the pore wall $[10,11]$, we obtain an expression for the contact angle as

$$
\cos \phi=\frac{W_{p s}}{\sigma_{s l}}-1
$$

The Gibbs-Thomson equation may also be written in a simpler form [19]:

$$
\Delta T_{m}=\frac{k_{G T}}{x}
$$


where $x$ is a dimension that defines the effective size of the pore and $k_{G T}$ is a constant that depends on the liquid, the pore geometry and the wetting nature of the pore walls.

More specifically [20],

$$
\Delta T_{m}=\frac{k_{G T}}{x}=\frac{k_{g} k_{s} k_{i}}{x}
$$

where, $k_{g}$ is a geometric constant dependent on the interfacial shape [21], $k_{s}=\frac{T_{m}^{\infty}}{\Delta H_{f} \rho_{s}}$ is a constant specific to the solid crystal thermodynamic parameters, and $k_{i}=\sigma_{s l}-W_{p s}$ is a constant specific to the two inter-surface interaction terms of the crystal.

\subsection{The geometry term in the Gibbs-Thomson equation.}

The factor of ' 4 ', in both the Gibbs-Thomson equation (Eq. 4) and the Kelvin equation is the manifestation of the $k_{g}$ term, and is only appropriate to a hemispherical crystalline-liquid interface in cylindrical geometry. It is currently believed that the structural geometry factor $k_{g}$ (at least for the freezing branch of the isobars) is a function of the pore surface area to volume ratio $s_{p} / v_{p}[21,22]$. It is important to note that the $k_{g}$ term also applies to the Kelvin equation and its derivatives such as the Barret, Joyner and Halenda (BJH) analysis [23].

A version of the Gibbs-Thomson equation has been derived [21], that does not assume $\Delta T_{m} \ll T_{m}$, and that also relates the geometric term $k_{g}$ to the pore surface-area to volume ratio $s_{p} / v_{p}$. This full version has a logarithmic form very similar to that of the Kelvin equation :

$$
\Delta H_{f} \rho_{s} \ln \left(\frac{T_{m}(x)}{T_{m}^{\infty}}\right)=-\sigma_{s l} \frac{s_{p}}{v_{p}}(x) \cos \phi
$$


Theoretical analysis [21, 22] suggests that for the melting event the theoretical ratio for $k_{g}^{\text {sphere }} / k_{g}^{\text {cylinder }}$ may be either $3 / 2$ or 2 , depending on the models used. From NMR cryoporometry calibration experiments (see Sections 4. 4.4) it would appear that the measured ratio for $k_{G T}^{s o l-g e l} / k_{G T}^{c y l}$ is close to $1 / 2$ for agreement with gas adsorption results. That the $k_{g}$ term also applies to the Kelvin equation may be an explanation for the above reversal of this ratio, as these ratios are based on gas adsorption calibrations.

In Section 4.3 and the explicit neutron diffraction cryoporometry results for the geometry term ratio for freezing-melting (Section 4.4) it appears that the measured ratio for $\Delta T_{f} / \Delta T_{m}$ in cylindrical pores is close to $6 / 4$, for agreement with gas adsorption results, which is consistent with Eq. 8.

\subsection{Second order (linearity) terms in the Gibbs-Thomson equation.}

Non-linearity in the Gibbs-Thomson equation was observed in early work when using cyclohexane as the probe liquid in sol-gel silicas [24], and was also reported for various systems including water and benzene in mesopores [25] and is now ascribed to a layer with enhanced rotational motion [20].

Thus for a surface layer of thickness $\varepsilon$ in a pore of diameter $x$

$$
\Delta T_{m}=\frac{k_{G T}}{x-2 \varepsilon}
$$

However the main problem with such a simple description is that in some systems such as cyclohexane the value for $\varepsilon$ appears to depend on the time $2 \tau$ at which the magnetization decay is sampled [24], such that, for $\tau>\tau_{c}$, $\varepsilon \Rightarrow 0$. The explanation for this appears to again reside with the properties of the layer with enhanced rotational motion; experimental results are discussed 
in Section 4.2, and further results for the properties of the layer are discussed in Section 5.

\section{The application of NMR relaxation, diffusion and cryoporome- try techniques to the study of the properties of liquids in pores.}

There have been a wide range of nuclear magnetic resonance investigations into the properties of water/ice systems in confined geometry, using the following five main techniques.

1. Measurement of changes in the line-shape of the water proton resonance due to the magnetic susceptibility field gradients that are present in porous systems [26, 27, 28, 29, 30, 31, 32]. In high NMR static magnetic fields, diffusion in these gradients may dominate the NMR relaxation of liquids in pores.

2. Measurement of changes in the $T_{1}$ (longitudinal or spin-lattice), $T_{2}$ (transverse or spin-spin) and $T_{1 \rho}$ (rotating frame) relaxation times which is caused by exchange of magnetisation between the liquid in the pore and the pore wall $[33,34,35,36,37,38,39,27,40,41,42$, $43,44,45,46,47]$. In low NMR static magnetic fields, this exchange of magnetisation tends to dominate the NMR relaxation times of liquids in pores.

3. Measurement of the variation of the amplitude of the liquid component in the $T_{2}$ relaxation curve with temperature, and the interpretation of this in terms of the Gibbs-Thomson relationship, which is known as NMR cryoporometry $[19,48,49,50,37,24,51,41,42,52,22,53,54$, $55,56,57]$. 
4. Measurement of translational diffusion of the confined water using pulsed magnetic field gradient or fringe magnetic field gradient techniques $[58,59,60,61,62,63,64,65,66,67,68,69,70,71,72,73]$.

5. Direct measurement of nuclear magnetization transfer between the liquid in the pore and the pore wall, as a function of time $[74,64,75,76]$.

Most of these techniques are to some degree equivalent in that they monitor the dynamical processes by measuring the transfer of nuclear spin magnetization and thus the coupling between different parts of the system being studied. A particularly significant case is the examination of magnetisation transfer in systems consisting of water, ice and the containing silica surfaces, as mediated by both rotational and translational diffusion and modified by re-orientational averaging. As a result, these techniques are highly sensitive to the state of the water/ice at the silica surfaces, which may be in a significantly different thermodynamic state to that of bulk water/ice. Both relaxation and diffusion measurements in particular point to the existence of mobility in water/ice systems in pores.

The prime features dominating the measured NMR relaxation are well understood but further developments will be needed in order to relate the details in individual confined geometry systems to the basic physical processes. The direct measurement of the magnetisation transfer is proving highly informative for the study of liquid-substrate interactions in porous media. NMR cryoporometry is unique in not only being reliant on changes in the NMR relaxation, but also in providing information from the Gibbs-Thomson Free Energy in the system. NMR cryoporometry is proving invaluable for its ability to localise components of the liquids and their crystals to the interior or 
exterior of the pores, by application of the Gibbs-Thomson equation.

\section{NMR Cryoporometry : experimental.}

A system consisting of water/ice in the highly regular templated SBA15 porous silica TLX-1-5 (F5) [77] is considered. This system consists of cylindrical pores of about $86 \AA$ diameter on a hexagonal lattice.

An NMR pulse sequence of $90^{\circ}-\tau-180^{\circ}-\tau$ - echo is used to monitor the quantity of mobile liquid in the pores, at a time when the signal from brittle ice will have decayed, but that from the liquid will be substantially intact. If the water in the pores is all frozen, and then the system is warmed up at a slow rate, and the NMR echo amplitude is monitored at a time of $2 \tau=2 \mathrm{~ms}$, then for a highly over-filled sample (excess water around the grains) the results in Fig. 1 are obtained. The step (D to C) at about $-13^{\circ} \mathrm{C}$ corresponds to the melting of the liquid in the pore at the Gibbs-Thomson reduced temperature, that is, the increase in the Gibbs Free Energy due to being confined within these pores is dynamically equivalent to a thermodynamic temperature increase of about $13^{\circ} \mathrm{C}$. There is then a plateau $(\mathrm{C}$ to B) where no further liquid melts, followed by another step (B to A) at the normal bulk melting point when the liquid outside the grains melts.

The step corresponding to the melting of the liquid in the pores may then be differentiated and transformed, [49] to give a pore size distribution, Fig. 2 . 


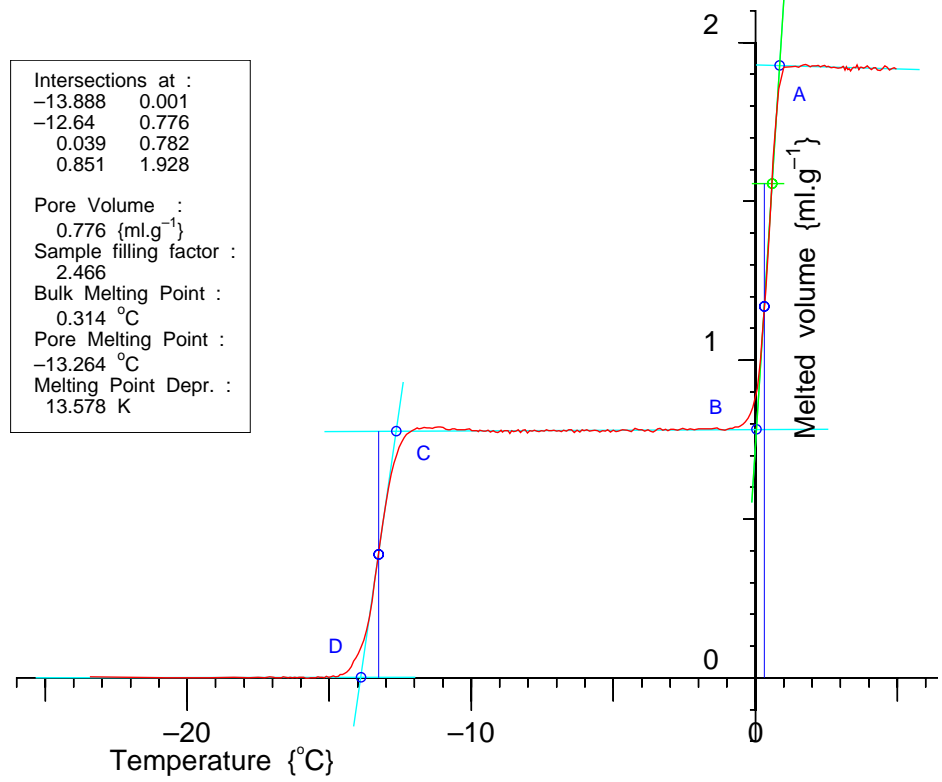

Figure 1: NMR Cryoporometry melting curve for water in SBA-15 (F5) silica, at a warming rate of $0.05 \mathrm{C}^{\circ} \cdot \mathrm{min}^{-1}$, with linear fitting to graph sections and hence extraction of phase transitions and pore volume. Straight-line fits are made to each linear section of the data, and their intersections are at points A to D: All the liquid is frozen below D; then from D to $\mathrm{C}$ the liquid in the pores melts; on the plateau from $\mathrm{C}$ to $\mathrm{B}$ no further liquid melts; from B to A the bulk liquid outside the grains melts: above A all the liquid is melted. Thus knowing the mass of all the liquid in the sample (and assuming the density), the ratio of the amplitudes B/A gives the total pore volume. The difference in temperature between B,A and D,C gives the melting point depression in the pores [24]. Graph reproduced with permission from Elsevier [78]. 


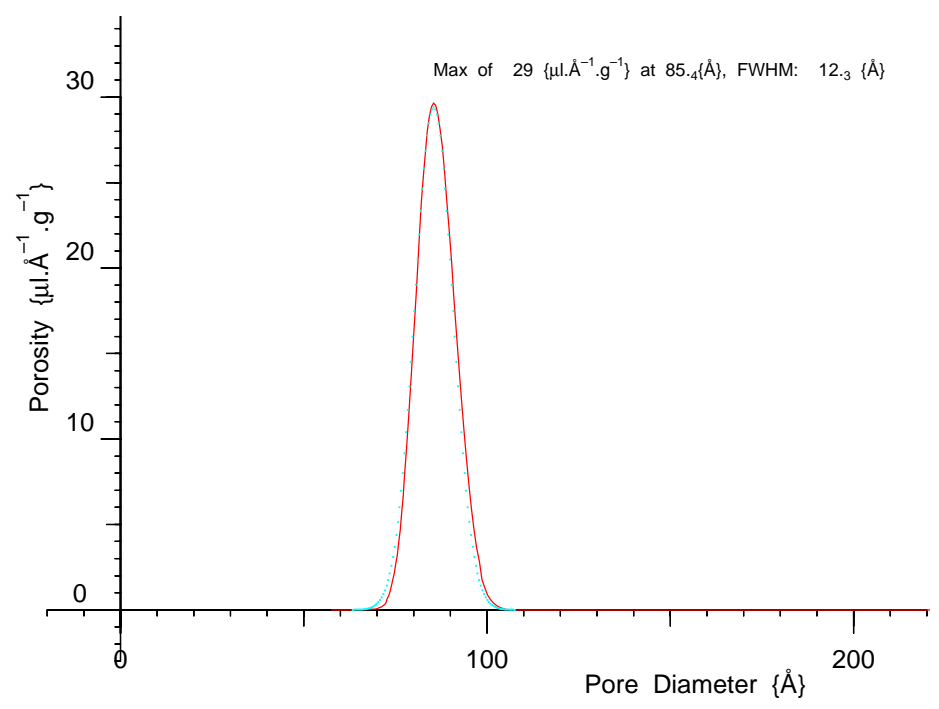

Figure 2: Pore diameter as measured by NMR Cryoporometry using water in SBA-15, assuming a cryoporometric constant $k_{G T}$ twice that appropriate to sol-gel silicas. Line: measured data; dots: fitted Gaussian, with a peak porosity of $29 \mu \mathrm{l} \AA^{-1} \mathrm{~g}^{-1}$ at a pore diameter of $85.4 \AA$ and full width at half maximum (FWHM) of $13.3 \AA$. Graph reproduced with permission from Elsevier [78]. 
4.1. NMR Cryoporometry : experimental calibration of $k_{G T}$ for the melting transition.

By measuring the median of the melting point depressions for water in sol-gel silicas with a range of pore diameters, as calibrated by gas adsorption, and by plotting against inverse pore diameter, a reasonable straight-line fit is obtained, see Fig. 3, from which one may deduce a value for the GibbsThomsom Coefficient $k_{G T}^{\text {sol-gel }}$ for water of about 58.2 K.nm (for the geometry applicable to sol-gel silicas).

To obtain a correct value for $k_{G T}$ for a brittle-ice to water transition by NMR one must measure at a Free-Induction-Decay (FID) or echo sampling time in excess of $1 \mathrm{~ms}$. For the work relating to Fig. 3, times of $4 \mathrm{~ms}$ to $20 \mathrm{~ms}$ were used and were shown to give equivalent results. The reason that measurements at shorter times give diverging results has recently become clear, and is discussed in Section 5, where evidence that water near an interface may enter a state with enhanced rotational motion is considered.

More recent work has returned significantly different results for $k_{G T}$ when measuring in templated silicas with cylindrical pores. This is expected, from the different values for the geometric term $k_{g}$. However it is important to obtain experimental values for $k_{g}$, and Fig. 4 shows a fit to data for three MCM-41 type silicas (+) [79], where the melting point depression is plotted against inverse pore diameter as measured by $\mathrm{N}_{2}$ gas adsorption using both Kruk-Jaroniec-Sayari (KJS) and density functional theory (DFT) analyses, and also a melting point depression for SBA-15 silica [78]. The melting point depression for SBA-15 data was measured with the same apparatus as shown in Fig. 2, the pore diameter being measured by $\mathrm{N}_{2}$ gas adsorption Frenkel- 
Halsey-Hill (FHH) analysis [78]. The best-fit straight line through the origin gives $k_{G T}^{c y l}$ for water as about 140 K.nm.

The melting points for a number of the sol-gel silicas used for Fig. 3 were re-measured on the same apparatus as for the SBA-15 data, using a $2 \tau$ acquisition time of between $2 \mathrm{~ms}$ and $10 \mathrm{~ms}$ (mostly $4 \mathrm{~ms}$ ). For nearly all the silicas $\mathrm{N}_{2}$ gas adsorption diameters were measured using Coulter SA 3100 instruments, and for many of the samples measurements were made on two separate instruments; measured values were not obtained for two of the silicas, and manufacturers' supplied pore diameters were used. The desorption BJH pore incremental volumes vs. pore diameter were peak fitted, and the medians of the desorption BJH pore cumulative volumes vs. pore diameter were also measured; both values were used to plot the measured melting point depressions (x) (Webber and Strange, unpublished work). The best-fit straight line through the origin gave $k_{G T}^{\text {sol-gel }}$ for water of about 58.0 K.nm, which is in reasonable agreement with Fig. 3. Given the scatter for both these data sets there appears to be little advantage in considering a surface layer : the minima in the $\Xi^{2}(\varepsilon)$ errors of the best-fits are fairly shallow.

In earlier work with only an estimate of $k_{G T}^{c y l}$ from the single SBA-15 sample [78] it was suggested that $k_{G T}^{c y l} / k_{G T}^{\text {sol-gel }}$ was about 2. Fig. 4 suggests that $k_{G T}^{c y l} / k_{G T}^{\text {sol-gel }} \sim 2.43$. This is significantly different from any theoretical value, but this may be due to the different analyses that have been applied to the $\mathrm{N}_{2}$ gas adsorption data, or may indicate the need for more consistent melting point data that have all been measured on the same NMR cryoporometric instrument. 


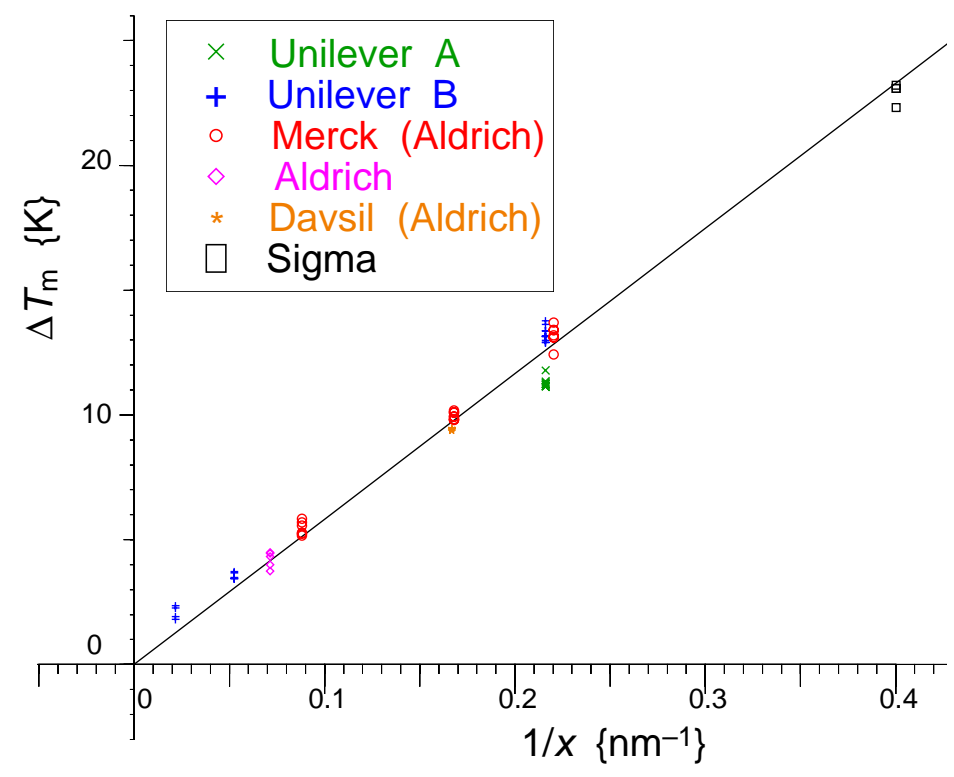

Figure 3: Median melting point depression $\Delta T_{m}$ for water in 10 different sol-gel porous silicas, from different sources, of diameter $25 \AA$ to $500 \AA$, as measured by an NMR $90^{\circ}-\tau$ $180^{\circ}-\tau$-Echo experiment, with a measurement time of $2 \tau=4 \mathrm{~ms}$, plotted against inverse nominal pore diameter $x$, as calibrated by gas adsorption. The slope of the best straight line fit to the 61 measurements, through the origin, gives $k_{G T}=58.2 \mathrm{~K} . \mathrm{nm}$. Graph reproduced with permission from Elsevier [51]. 


\section{Sol-Gel, MCM-41 + SBA-15 melting-point depressions}

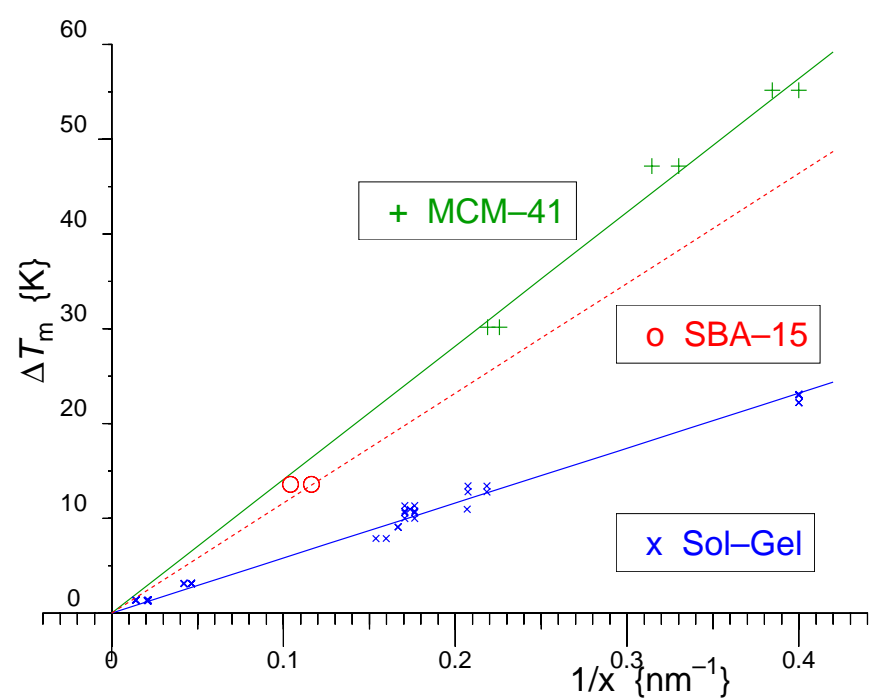

Figure 4: The relation of melting point depression $\Delta T_{m}$ to inverse pore diameter $x$, for water in various sol-gel silicas as calibrated by gas adsorption measurements (x) (data used with permission from Lab-Tools Ltd.), compared with melting point depressions for water in templated cylindrical pores : MCM-41 $(+)$ [79] (data used with permission from G.H. Findenegg) and SBA-15 (F5) (o) [78] (data used with permission from Lab-Tools Ltd.). The dotted line has twice the slope as the fit to the sol-gel silica data. 
4.2. NMR Cryoporometry : experimental effect of measuring time on the measured melting transition.

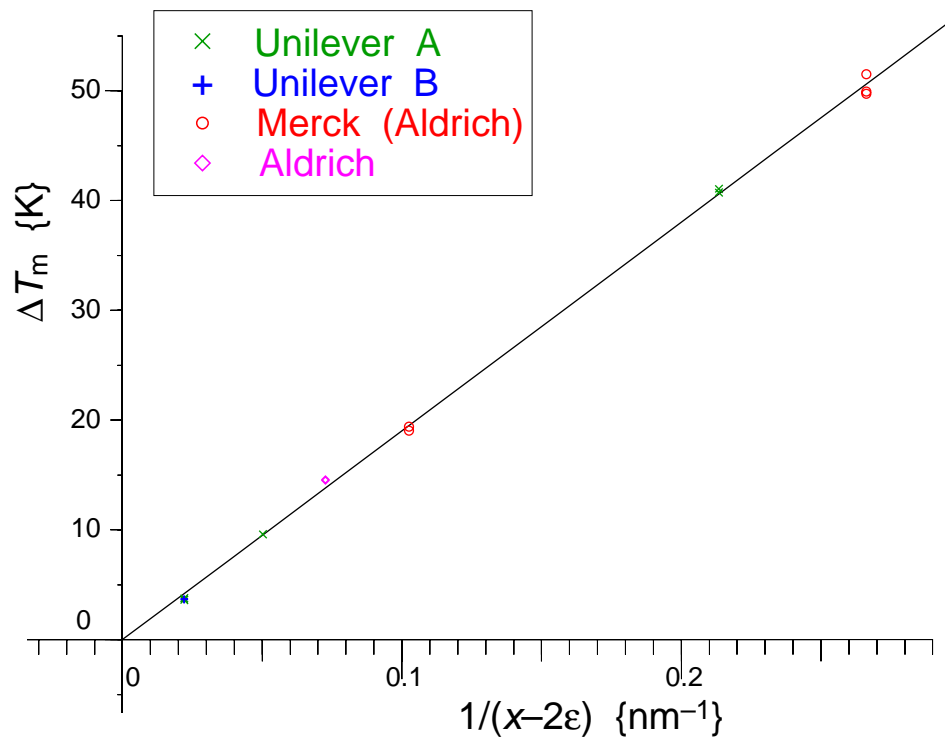

Figure 5: The melting point depression $\Delta T_{m}$ for cyclohexane in various sol-gel silicas of diameter $25 \AA$ to $500 \AA$, as measured by an NMR $90^{\circ}-\tau-180^{\circ}-\tau$-Echo experiment, with a measurement time of $2 \tau=20 \mathrm{~ms}$, plotted against inverse nominal pore diameter $x$, as calibrated by gas adsorption. The slope of the best straight line fit to the 14 measurements, through the origin, gives $k_{G T}=190.1$ K.nm, assuming a surface layer term $\varepsilon=0.121 \mathrm{~nm}$. Graph reproduced with permission from Elsevier [80].

The presence of rotational motion is well known in solid cyclohexane, which has a plastic phase between the brittle and liquid phases, and similar plots for multiple pore diameter silicas, as a function of pore diameter, for cyclohexane, such as Fig. 5, are shown to require a surface layer term $\varepsilon$ to be included in the Gibbs-Thomson equation (i.e. to obtain a straight line in plots such as Fig. 5,) as discussed in Section 2.2. The constants required 
for these fits (Table 1) show that the measured surface layer thickness $\varepsilon$ is $\tau$ dependent [24].

\begin{tabular}{|c|cc|}
\hline $2 \tau$ & $k_{G T}$ & $\varepsilon$ \\
$\{\mathrm{ms}\}$ & $\{\mathrm{K} \AA\}$ & $\{\AA\}$ \\
\hline 4 & 2419 & $4.47_{6}$ \\
10 & 2032 & $3.40_{7}$ \\
20 & 1901 & $1.21_{4}$ \\
40 & 1789 & $0.00_{0}$ \\
\hline
\end{tabular}

Table 1: Slope $k_{G T}$ of melting point depression, and surface layer $\varepsilon$, for cyclohexane in sol-gel silica, measured at time $2 \tau$ [24].

Fig. 7 indicates that for cyclohexane, for measurements with $2 \tau \geq 26$ ms the mvalue of the pore diameter derived from eq. 9 will be independent of $\tau$, consistent with the standard minimum value of $\tau=20 \mathrm{~ms}$, used for many years in cryoporometric measurements on cyclohexane. Fig. 7 implies that for the first $5.4 \AA$ there is something different about the properties of cyclohexane within pores. This variation is now believed to be due to a surface layer with enhanced rotational motion, whose thickness depends on temperature, as discussed in Section 5.

\subsection{NMR Cryoporometry : experimental calibration of $k_{G T}$ for the freezing} transition.

NMR Cryoporometry conventionally uses the equilibrium melting transition, as for many liquids in pore systems on a simple cooling the liquid will enter a non-equilibrium super-cooled state, before finally freezing at a 


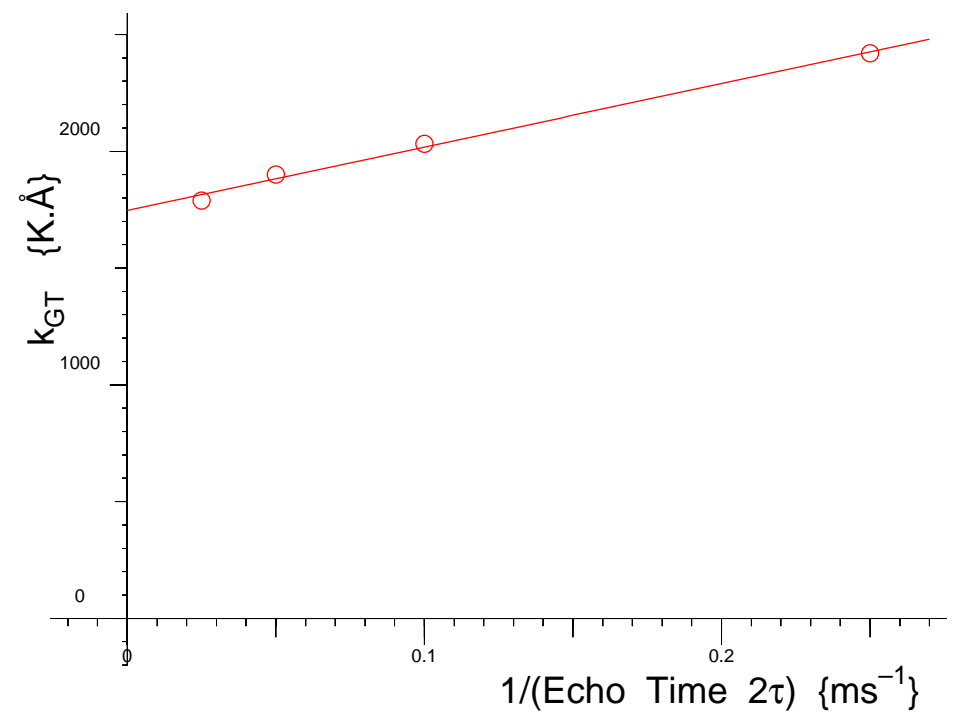

Figure 6: Measured Gibbs-Thomson coefficient $k_{G T}$ for cyclohexane in sol-gel silica as a function of inverse measuring time $2 \tau$ (Re-plot of data from Table 1) [24]. Graph reproduced with permission from Lab-Tools Ltd.

temperature that does not correctly encode the Gibbs-Thomson coefficient.

Recently a multi-ramp measurement technique to also observe an equilibrium freezing transition such as is shown in figures 8, 9 has been reported independently by Petrov and Furo [81] and Webber et al. [82, 83].

The sample is initially cooled through the supercooling event until all the liquid is frozen, then it is warmed until the liquid in the pores is melted, but with the liquid around the grains remaining frozen. During a subsequent cooling ramp, the ice around the grains serves to initiate freezing inside pores at the true Gibbs-Thomson equilibrium freezing point. A final warming ramp again melts the liquid in pores and then finally the liquid around the grains. The latter event serves as a reference temperature for the bulk melting point, helping remove zeroth order instrumentation offsets. 


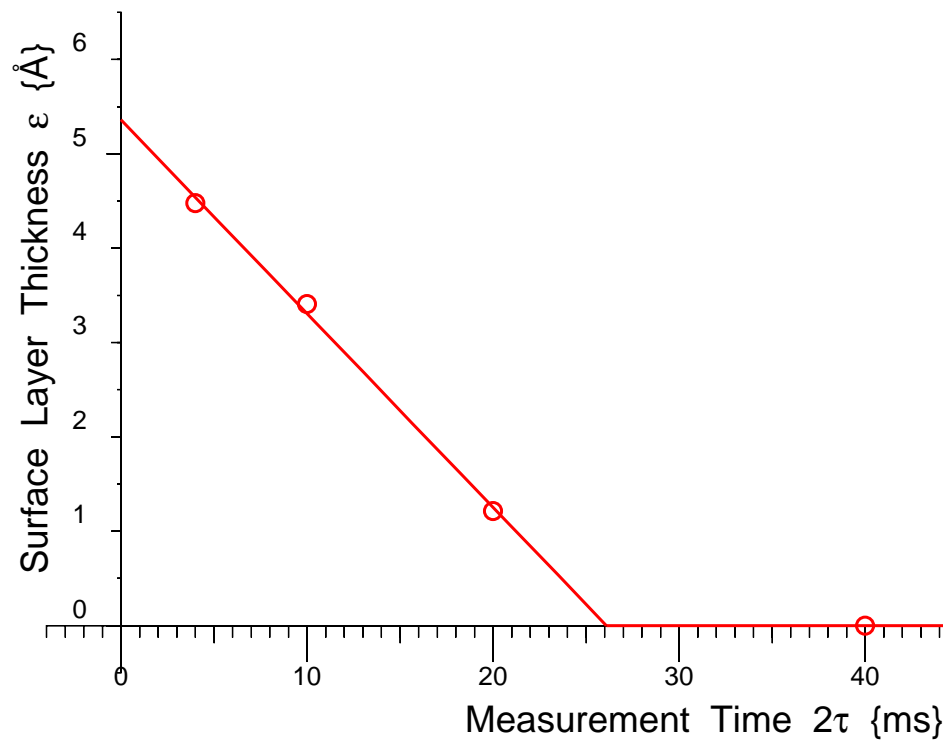

Figure 7: Measured surface layer term $\varepsilon$ for cyclohexane in sol-gel silica as a function of measuring time $2 \tau$. For $2 \tau \geq 26 \mathrm{~ms}, \varepsilon=0$ (Plot of data from Table 1$)$. Graph reproduced with permission from Lab-Tools Ltd. 


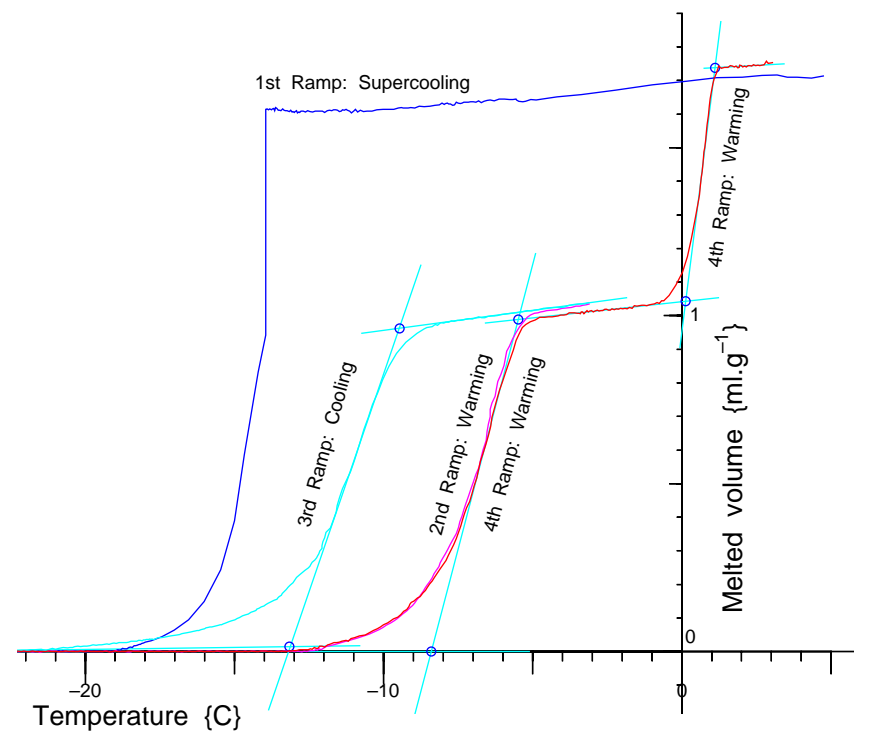

Figure 8: Multi-ramp NMR Cryoporometry measurements on water in $10 \mathrm{~nm}$ nominal pore diameter sol-gel silica, probing the freezing event as well as the melting events [82]. Graph reproduced with permission from Elsevier [83]. 


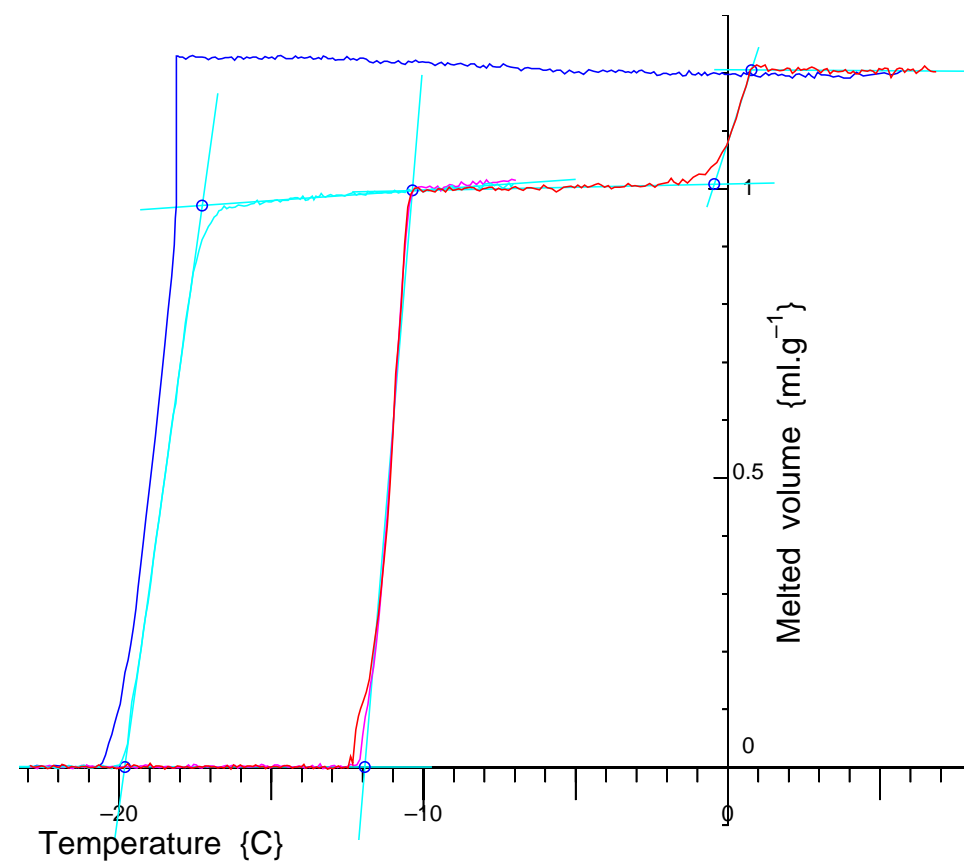

Figure 9: Multi-ramp NMR Cryoporometry measurements on water in SBA-15 silica, probing the freezing event as well as the melting events [82]. Graph reproduced with permission from Lab-Tools Ltd. 
This freezing event is at a lower temperature than the melting event; this may be due to the geometry of the freezing meniscus (a hemisphere growing into the pore) being different from the melting meniscus (which is cylindrical in the case of cylindrical pores), though other effects may also be present in certain materials, including the effect of restricted access in "ink-bottle" type pore geometry $[84,85,86,79]$.

\subsection{Effects of interface geometries on freezing/melting behaviour : experi- mental.}

Measuring the freezing and melting transitions at a few warming-rates, and then extrapolating to zero warming rate, removes any first order error, and reduces the remaining error in the measured freezing and melting points to second order.

For multi-ramp NMR Cryoporometry on the above SBA-15 (F5) sample the best estimate to-date, after extrapolation to zero warming-rate, gives $\Delta T_{f} / \Delta T_{m}=1.45 \pm 0.05$. This suggests that the ratio for this pore geometry may actually be close to 1.5; if we assume that the advancing ice freezingfront in the cylindrical pores is spherical, while the ice melting-front geometry is cylindrical, Eq. 8 would indeed predict such a ratio.

There is a detailed discussion of the $\Delta T_{f} / \Delta T_{m}$ ratio, with proposed equations, and with experimental results for water, benzene, cyclohexane and cyclooctane in $23.7 \mathrm{~nm}$ and $72.9 \mathrm{~nm}$ pore diameter CPG silicas, provided by Petrov and Furo [22]. 


\section{Water/ice at an interface : Plastic Ice.}

\subsection{Water/ice at a silica interface}

A system consisting of water/ice in a cylindrical pore templated SBA-15 porous silica [77] has been probed using neutron diffraction, neutron diffraction cryoporometry, NMR transverse relaxation and NMR cryoporometry techniques $[2,83,20,78,87,88]$.

The first clues that there were processes at work in the pores, beyond standard Gibbs Thomson melting point depression, were given by a novel analysis of the neutron diffraction data.

\subsubsection{Structure, as determined by Neutron diffraction cryoporometry, using} a global analysis, with a fully-filled sample

Diffraction curves were measured for $\mathrm{D}_{2} \mathrm{O}$ vs. scattering vector $q$, in a range of templated samples, as a function of temperature, and for various filling factors; we shall discuss the measurements on the SBA-15 TLX-1-5 (F5) sample for filling factors $\mathrm{f}=1.31,0.95$ and 0.61 .

Fig. 10 shows a selection of neutron scattering data sets for an over-filled sample (i.e. with excess $\mathrm{D}_{2} \mathrm{O}$ around the grains), measured on a warming ramp, plotting every tenth diffraction curve. At the highest temperatures there is a broad liquid-water peak, at lower temperatures the signal shows the hexagonal ice melting to water, and at yet lower temperatures there is a complex inter-conversion between defective cubic ice, hexagonal ice and a water-like component.

Neutron diffraction cryoporometry (NDC) [78] is a recently developed technique that uses such data to present plots of the quantities of each of 
the water/ice phases in the system being studied, as amplitude plots, in a similar manner to NMR cryoporometry (NMR-C), introduced by Strange et al $[19,56]$, which in turn is a development of the gas adsorption $[89,23]$ and differential scanning calorimetry (DSC) thermoporosimetry [90] techniques.

This data set was then globally fitted using the templates shown in Fig. 11 to extract the amplitudes of the water, hexagonal ice and cubic ice components. The paper describing the NDC technique [78] concluded with the statement that the technique needed further development so as to return normalised amplitudes. It is believed that a reasonable approach to this normalisation has now been developed.

The normalised measured neutron diffraction cryoporometric curves are shown for the above components in Fig. 12. In this graph the total of the displayed quantities for each of the three phases in the sample, water, cubic ice and hexagonal ice, at any particular temperature, sum to unity. An important feature is that the curve for water can be seen to have a significant component well below the main melting event in pores,

The hexagonal ice can be seen to mainly melt at temperatures appropriate to bulk $\mathrm{D}_{2} \mathrm{O}$ ice (allowing for instrumental effects), indicating that it is external to the pores, while the cubic ice melts at a lowered temperature appropriate to the Gibbs-Thomson melting point reduction in these pores. Thus the ice forming in these pores is mainly cubic, in accordance with previous studies on sol-gel silicas with pores $<30 \mathrm{~nm}$ diameter. However, a further $10 \%$ of the hexagonal ice is also seen to form at a reduced temperature, indicating that some of the ice in the pores of the SBA-15 incorporates a hexagonal component. 


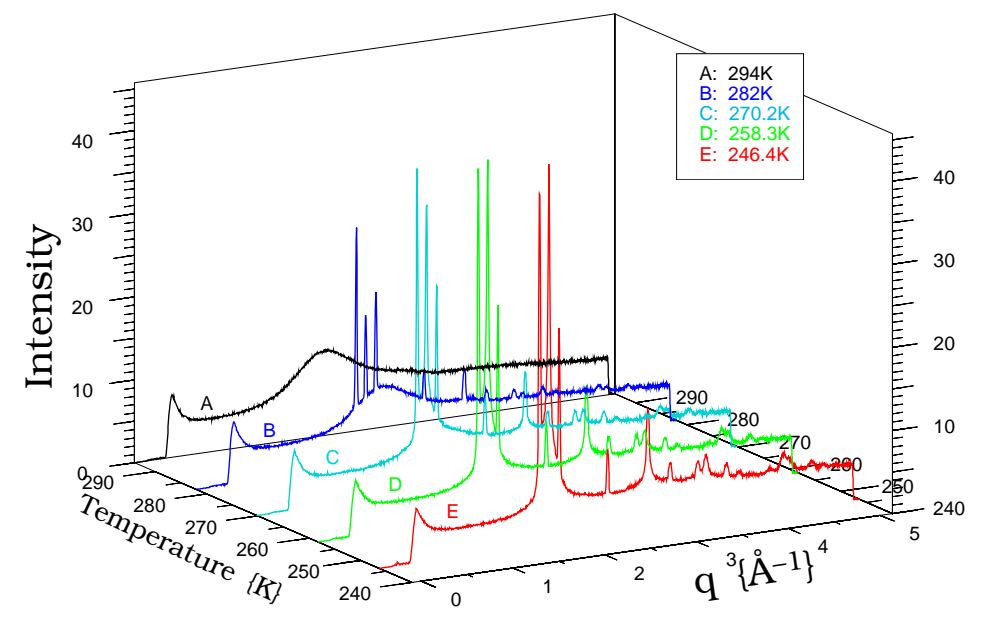

Figure 10: The diffraction pattern for water/ice in an over-filled sample of SBA-15 silica (F5), of 8.6nm pore diameter, as a function of temperature during a warming ramp. Graph reproduced with permission from Institute of Physics [91].

The data clearly also shows a disordered component, that is best matched by the water template. However a study of the actual diffraction data shows a difference from the bulk water curves. This component exists below the normal Gibbs-Thomson depressed temperature for $\mathrm{D}_{2} \mathrm{O}$ water in these pores, extending a further 50 to $80 \mathrm{~K}$, down to around or below $200 \mathrm{~K}$.

As the temperature is further lowered, the amount of the disordered component decreases, converting primarily to further cubic ice, but some clearly also converts to hexagonal ice. As the temperature is again increased, the crystalline ices reversibly transform back to the disordered component, indicating a surprising equilibrium between the phases. The dynamics of this disordered component was then investigate by NMR relaxation; this will be discussed in the next section.

Fig. 12 is a complex graph, and further features will be discussed later in 


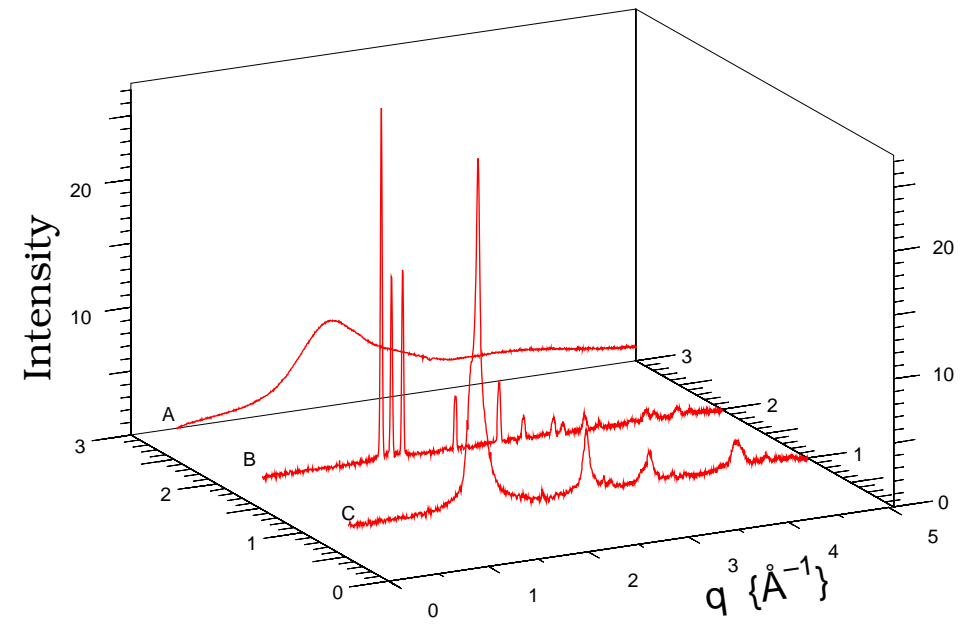

Figure 11: Neutron diffraction template curves for three ice/water phases: A) water, B) hexagonal ice, C) defective cubic ice. Graph reproduced with permission from Elsevier [78].

Section 5.2.2. Fig. 13 shows a normalised graph for the phase fractions for the SBA-15 pores nearly filled with $\mathrm{D}_{2} \mathrm{O}(\mathrm{f}=0.95)$. In this graph the total of the displayed quantities for each of the three phases in the sample, water, cubic ice and hexagonal ice, at any particular temperature, again sum to unity. A very significant feature of this graph is that nearly all the water in the pores converts to cubic ice, with only $1 \%$ hexagonal ice. It has been known for some time that the water in pores less than about $30 \mathrm{~nm}$ in diameter tends to form cubic ice, but these fractional plots are very enlightening : this data will be discussed further in the section on partial filling. 


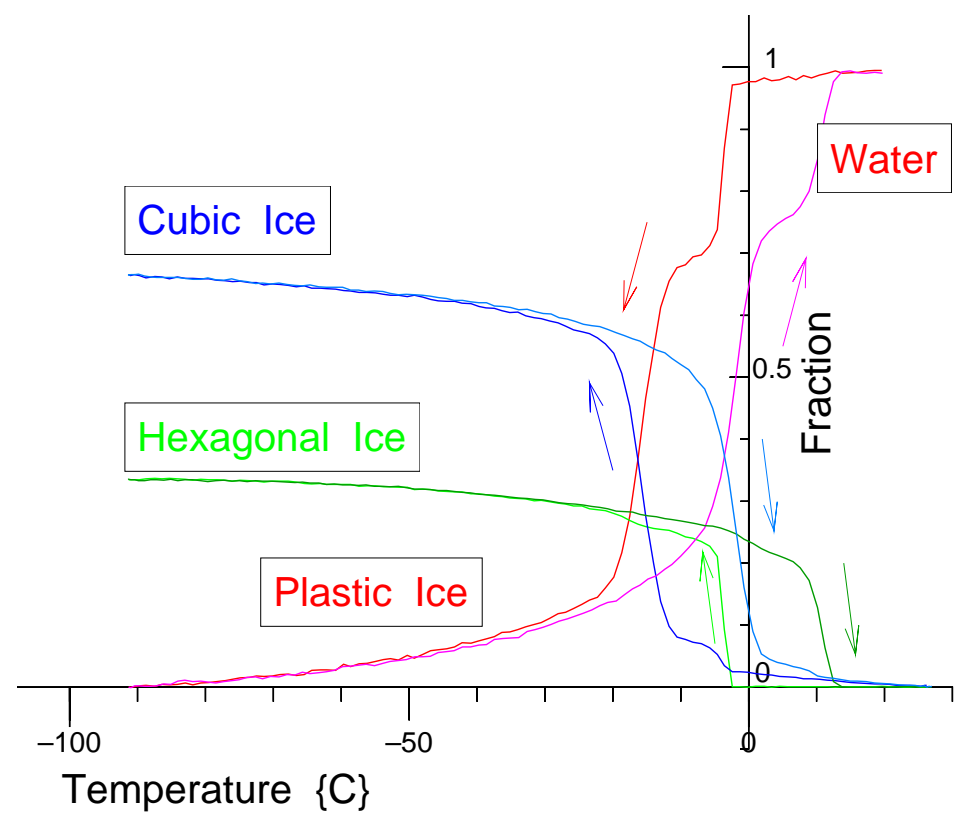

Figure 12: Normalised Neutron diffraction cryoporometric curves for ice/water freezing and melting in over-filled SBA-15 (F5) templated silica ( $\mathrm{f}=1.31$ ) with a nominal pore diameter of $86 \AA$, showing the separately resolved data for cubic ice, hexagonal ice and water. Based on a prior non-normalised graph, reproduced with permission from LabTools Ltd. [20].

\subsubsection{Dynamics, as determined by NMR transverse relaxation, for plastic} ice in a fully-filled sample.

NMR $T_{1}$ and $T_{2}$ measurements were made on an over-filled sample $(\mathrm{f}=1.20)$, which, below the main freezing event in the pores, showed a two component signal, with one component being brittle ice with a $T_{2}$ value of around 10 $\mu \mathrm{s}$, the other consisting of a much longer exponential (Fig. 14). This longer component had a value of $T_{2}$ that showed a plateau as a function of temperature of around 100 to $200 \mu \mathrm{s}$, becoming more brittle-ice like at around $-80^{\circ} \mathrm{C}$, see Fig. 15. The amplitude of this component also decreased progressively 


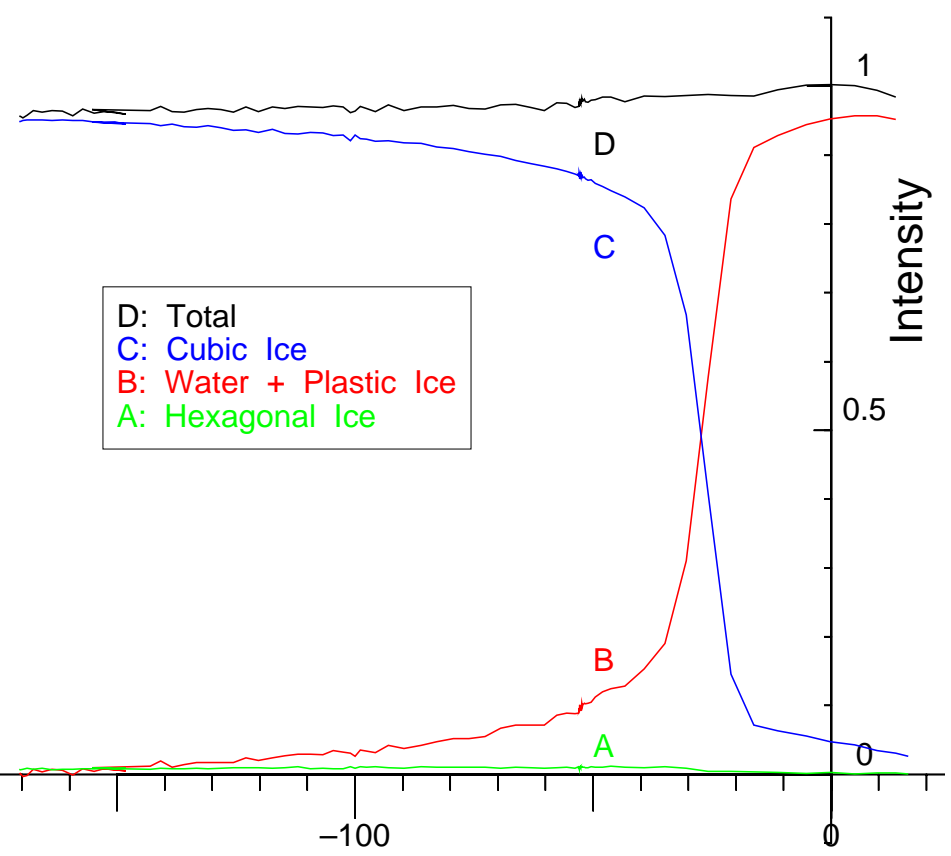

Temperature $\{\mathrm{C}\}$

Figure 13: Normalised Neutron Diffraction Cryoporometry graphs of $\mathrm{D}_{2} \mathrm{O}$ water / ice fractions in SBA-15 templated silica : Nearly filled, $\mathrm{f}=0.95$, for a cooling ramp. Graph reproduced with permission from American Institute of Physics [92]. 
and reversible at low temperatures, see Fig. 16.

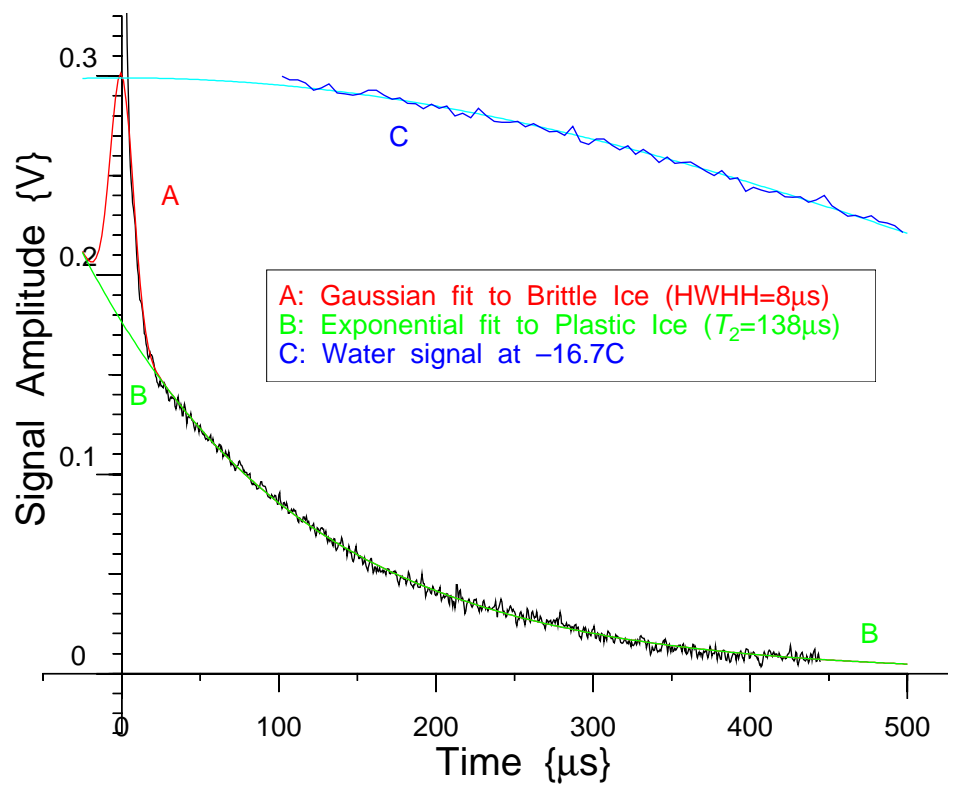

Figure 14: Free Induction Decay data for $\mathrm{H}_{2} \mathrm{O}$ in SBA-15 f=1.20 at $-20 \mathrm{C}$, showing brittle and plastic ice. (Boltzmann and Q corrected). Graph reproduced with permission from Lab-Tools Ltd. [93].

The NMR $\mathrm{T}_{2}$ data for this longer relaxation component in an over-filled sample $(\mathrm{f}=1.20)$ is plotted against inverse temperature as an Arrhenius graph in Fig. 15.

The activation enthalpy for the sloping part of cooling ramp over the temperature range $180 \mathrm{~K}$ to $230 \mathrm{~K}$ was determined as $4.6 \mathrm{~kJ}$ mole ${ }^{-1}$. This is the is believed to be due to the on-set of rotational motion.

At higher temperatures there is the plateau that is believed to relate to the dynamical region where rotational motion is probably still increasing, but where, for the purposes of NMR relaxation, the effective proton separations just average to a constant value equal to the molecular-centre separations 


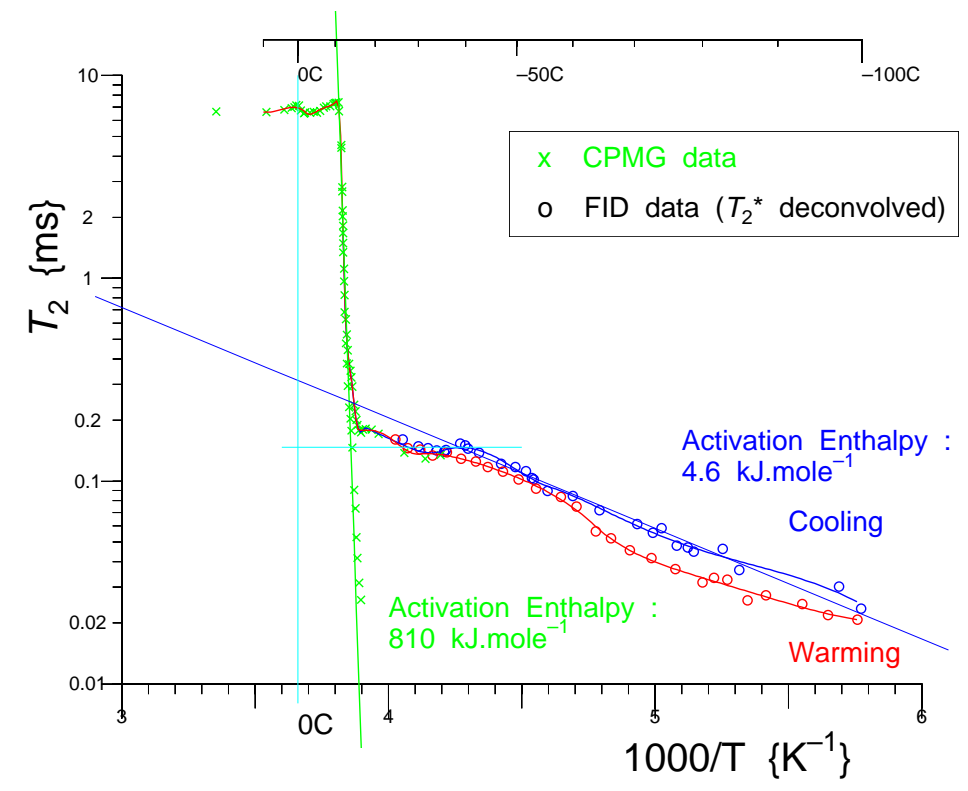

Figure 15: Variation with temperature of the NMR long $T_{2}$ relaxation time component for plastic ice in SBA-15 porous silica, shown as an Arrhenius plot, for overfilled pores with a filling factor $f=1.20$. Graph reproduced with permission from Institute of Physics [20]. 


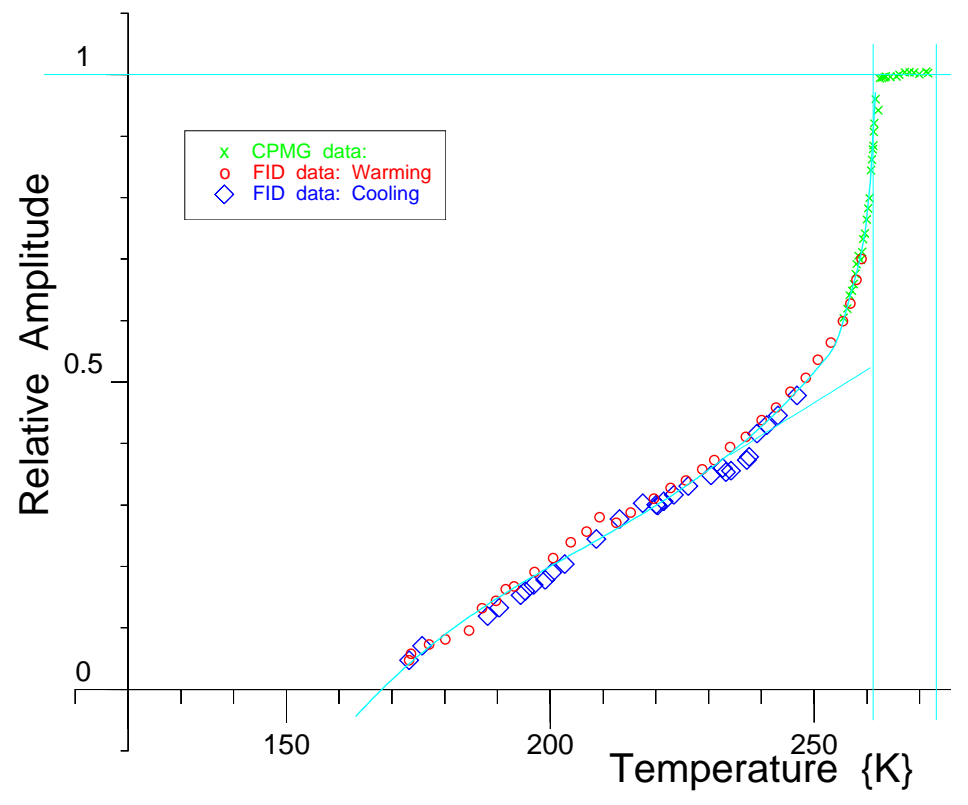

Figure 16: Amplitude of NMR signal for plastic ice in SBA-15 (F5). Maximum plastic ice fraction to total water-ice in pore $=0.52$; then, for cylindrical geometry, max fractional thickness of plastic layer $=0.31$, which for $86 \AA$ pore diameter gives the maximum thickness for the layer of plastic ice as $13 \AA$. The thickness of this surface layer of plastic ice reduces with lowering temperature at a rate of $0.14 \AA . \mathrm{K}^{-1}$. Graph reproduced with permission from Institute of Physics [20]. 
[94]. There are various possible causes that need to be considered for this factor of 10 increase in the $\mathrm{T}_{2}$ at around $200 \mathrm{~K}$ to $220 \mathrm{~K}$, for the defective ice, over that for brittle ice as discussed previously $[2,83,20]$. These may be summarised as follows.

1. $\mathrm{T}_{2}$ relaxation in ice is due to the dipolar interaction between the protons, an increase in the actual distance between the protons will lengthen $\mathrm{T}_{2}$. Given the observed changes as a function of temperature in Fig. 15 it is improbable that this behaviour is due to a simple lattice expansion.

2. There may be translational diffusion, giving rise to motional narrowing. This may be expected to contribute to the observed $\mathrm{T}_{2}$, particularly given the large change in temperature and is the likely cause of the rapid change in $T_{2}$ in the vicinity of the melting point in the pores. However the observed behaviour of $\mathrm{T}_{2}(T)$ over the range $180 \mathrm{~K}$ to $250 \mathrm{~K}$ is not characteristic of a translational activation energy, as would be expected for translational motional narrowing.

3. There may be rotational diffusion. In plastic crystals, rotation is well known to give rise to a form of motional averaging such that the actual proton separations must be replaced by the separation of the molecular centres. This gives rise to a significant increase in $\mathrm{T}_{2}$ at the onset of rotational motion, followed by a plateau in $\mathrm{T}_{2}(T)$ as $\mathrm{T}$ increases [94].

The plot of $\mathrm{T}_{2}(T)$ in Fig. 15 is very similar to that for the plastic crystalline phases of materials such as cyclohexane, showing the form of the above case (3) with step and plateau, followed by the rapid rise of case (2) just before melting; consequently it is suggested that this longer ice component 
is in a plastic state and that this plastic ice corresponds to the disordered component as measured by neutron scattering.

Thus the increase in $\mathrm{T}_{2}$ in the $180 \mathrm{~K}$ to $230 \mathrm{~K}$ region is believed to be due to the onset of rotational motion, while for the plateau at about $0.004 \mathrm{~K}^{-1}$ the motional averaging effectively sets the proton-proton separation to be the same as the oxygen-oxygen separation. This gives rise to a significant increase in $\mathrm{T}_{2}$ at the onset of rotational motion, followed by a plateau in $\mathrm{T}_{2}(T)$ as $\mathrm{T}$ increases [94]. If this plateau is fitted with a constant amplitude, and this value is then subtracted from the $\mathrm{T}_{2}$ relaxation data on the Arrhenius graph, a further straight line is obtained. It is believed that this component represents the onset of translation motion, with a surprisingly large enthalpy of $810 \mathrm{~kJ} \mathrm{~mole} \mathrm{e}^{-1}$.

Fig. 16 shows data for the fractional amplitude of this longer relaxation component, with corrections for receiver non-linearity and also changes with temperature of the Boltzmann population and NMR coil quality-factor Q. These measurements indicate that the fraction of plastic ice in the pores increases from about 0.05 at $170 \mathrm{~K}$ to an extrapolated maximum of about 0.52 of the total volume at the melting point in the pores at about $260 \mathrm{~K}$. As this melting point is approached these amplitude data for the longer component also include the amplitude of any fraction with increased translational motion, and so increases rapidly. If one assumes that the plastic ice is in the form of a layer on the surface of cylindrical pores, then the maximum extrapolated fractional thickness is 0.31 . For $8.6 \mathrm{~nm}$ diameter pores this corresponds to a maximum layer thickness of about $1.3 \mathrm{~nm}$, decreasing at about $0.014 \mathrm{~nm} . \mathrm{K}^{-1}$ as the temperature is lowered. 


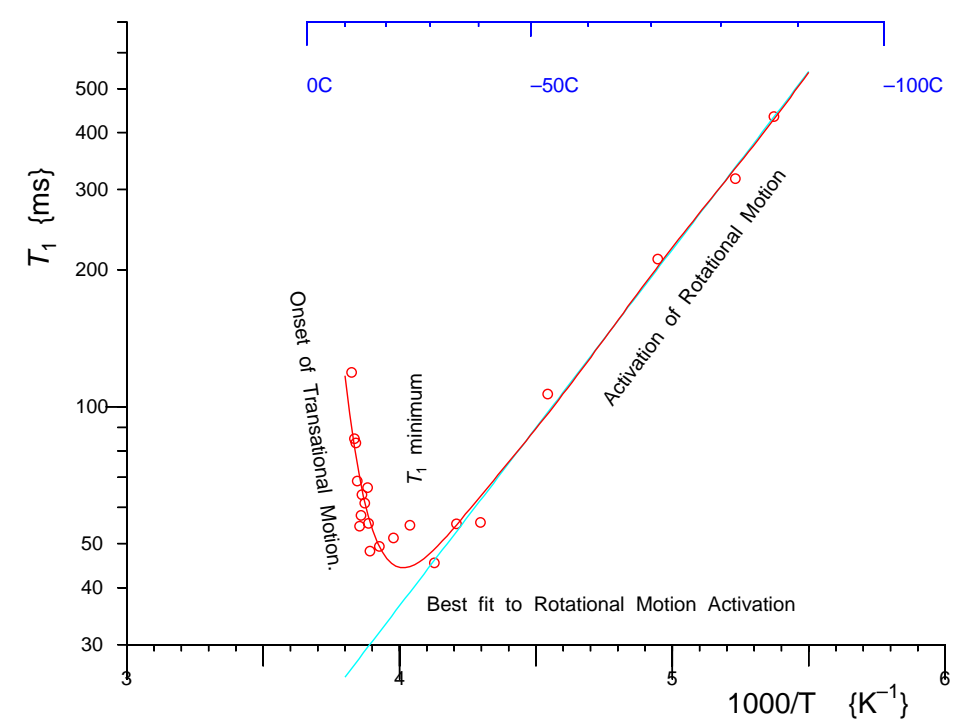

Figure 17: Variation with temperature of the $T_{1}$ of the NMR long $T_{2}$ relaxation time component for plastic ice in SBA-15 porous silica $-\mathrm{f}=1.20$. Graph reproduced with permission from Lab-Tools Ltd. [93]. 
The NMR $T_{1}$ relaxation behaviour (at $B_{0}=0.5 \mathrm{~T}$ ), for the longer $T_{2}$ relaxation component, is plotted against inverse temperature in Fig. 17. At low temperatures the rotational motion is minimal, and the $T_{1}$ value is long as would be expected. As the temperature is raised the rotation increases and $T_{1}$ decreases to a minimum. With the onset of translation, just below the melting event in the pores, motional narrowing sets in, and $T_{1}$ again increases.

Preliminary $T_{1}$ measurements as a function of $B_{0}$ have been made, but further analyses need to be performed on the data. It would be useful to also make $T_{1 \rho}$ measurements.

\subsection{Water/ice at a vapour interface}

NDC, NMR-C and NMR-R measurements have also been made on partiallyfilled pores, where there is also a water/ice to vapour interface. A hypothesis that has been generated to understand both the fully-filled and partiallyfilled pore NDC and NMR-C results, is that plastic ice forms at both the ice-silica and the ice-vapour interfaces, and that as the temperature is lowered, the plastic ice at the ice-silica interface converts to cubic ice, whilst the plastic-ice at the ice-vapour interface converts to hexagonal ice. This hypothesis is now tested against the measured results.

5.2.1. Dynamics, as determined by NMR transverse relaxation, for plastic ice in a partially-filled sample.

Early indication that the plastic ice component in partially-filled samples was larger and/or had a longer $T_{2}$ were given by the data in Fig. 18, where NMR-C measurements were performed on an un-modified sol-gel silica, a 
surface modified case of the same silica, and a partially-filled case of the latter, Jelassi, Castricum, Bellissent-Funel, Dore, Webber and Sridi-Dorbez, submitted. It can be seen that the signal below the main melting event in the pore has not relaxed to zero by the sampling time of $2 \tau=2 \mathrm{~ms}$ in the case of the surface modified silica, and that this is even more significant in the partially-filled case.

A study has been made of the $T_{2}$ behaviour of a partially-filled SBA-15 sample [92].

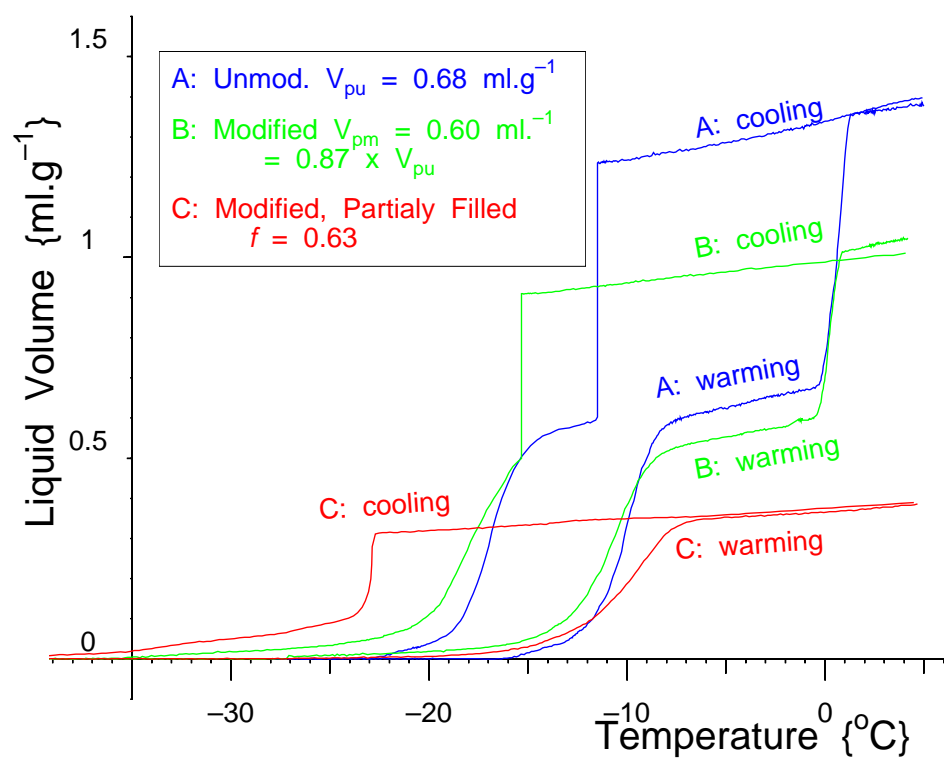

Figure 18: NMR cryoporometry curves for fully and partially-filled samples of a porous sol-gel silica, both with a hydrophylic surface and with a methylated hydrophobic surface [95], showing that the NMR long $T_{2}$ relaxation time component for plastic ice (below the pore melting/freezing events) is more significant in the partially-filled hydrophobic case, with filling factor $f=0.63$. Jelassi, Castricum, Bellissent-Funel, Dore, Webber \& Sridi-Dorbez, unpublished data [93]. 


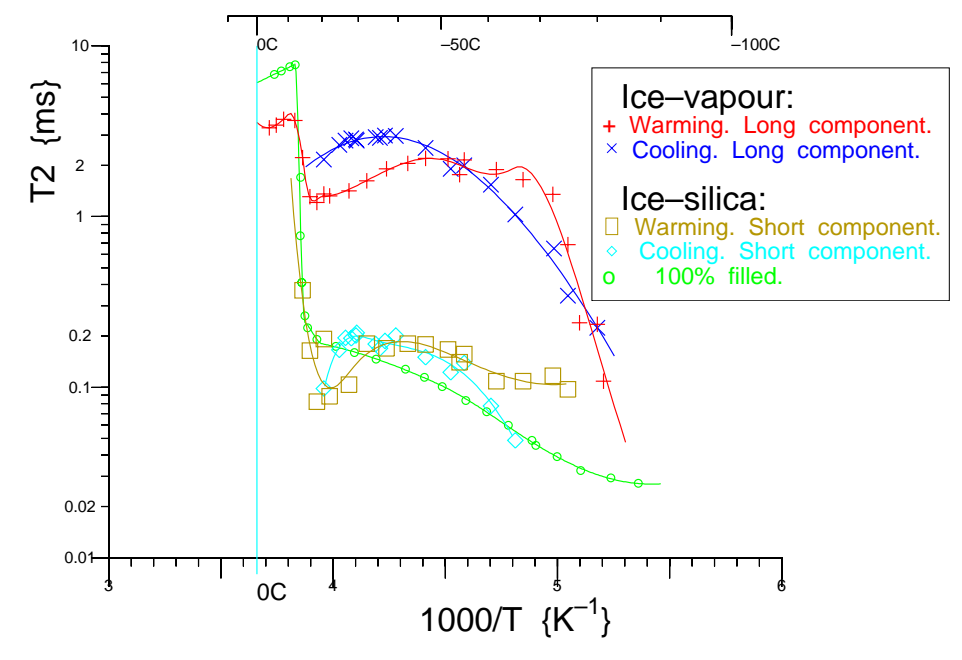

Figure 19: Variation with temperature of the NMR two intermediate $\mathrm{T}_{2}$ relaxation time components for ice at silica and air interfaces in SBA-15 porous silica - Arrhenius plot for filling factor $f=1.20$ (circles) and $f=0.50$ (remaining curves). Graph is an extension of prior work [92], and is reproduced with permission from Lab-Tools Ltd.

The NMR $\mathrm{T}_{2}$ relaxation data for a partially-filled sample $(f=0.50)$, that has ice-vapour interfaces as well as ice-silica interfaces, was found to have a short Gaussian component (corresponding to brittle ice) and two longer more exponential components. The $T_{2}$ relaxation times for the three components were found to differ by about an order of magnitude over most of the temperature range, and thus were cleanly separable. These relaxation times are plotted against inverse temperature as an Arrhenius graph in Fig. 19 with fully-filled data plotted for comparison (circles).

In the lower pair of traces the water-ice system can be seen to have very similar relaxation times to those of the water-ice system in the fully-filled case, where there is only an ice-silica interface. Thus this $T_{2}$ component is assumed to be plastic ice with a silica boundary. 
In the upper pair of traces the water-ice system can be seen to have relaxation times about 10 times longer than those of the water-ice system in the fully-filled case. Thus this $T_{2}$ component is assumed to be ice with a vapour boundary. The mobility is very high, so it not completely clear if one should consider this component as being ice. However there is clearly a further onset of translational motion just prior to the main melting event in the pores, and thus it is reasonable to again assume that there is a rotational mobility in the plateau region, such that on warming the system passes from a brittle ice state, through a plastic stage, before melting to a liquid state.

However this system needs to be more fully characterised by other techniques such as Quasi-Elastic Neutron Scattering (QENS), Pulsed Field Gradient NMR (PFG-NMR), as well as $T_{1}$ and $T_{1 \rho}$ relaxation measurements. QENS measurements performed on Vycor with a near monolayer coverage have been reported as showing a liquid-liquid transition [96]; however this work suggests that it is possible that one of these states is actually a plastic state.

\subsubsection{Structure, as determined by Neutron diffraction cryoporometry, using} a global analysis, with a partially-filled sample.

Neutron scattering measurements have been performed on partially-filled SBA-15 pores $(\mathrm{f}=0.61)$ [92]; the as measured data is shown in Fig. 20 [93]. A NDC analysis of this data (Fig. 21, [92]) shows that, on cooling, cubic ice and plastic-ice initially forms, but as the temperature is lowered, the plastic ice converts in approximately equal quantities to cubic ice and hexagonal ice. There is slightly less hexagonal ice than cubic ic (as would be expected for the smaller interfacial area at the ice-vapour interface compared with the 


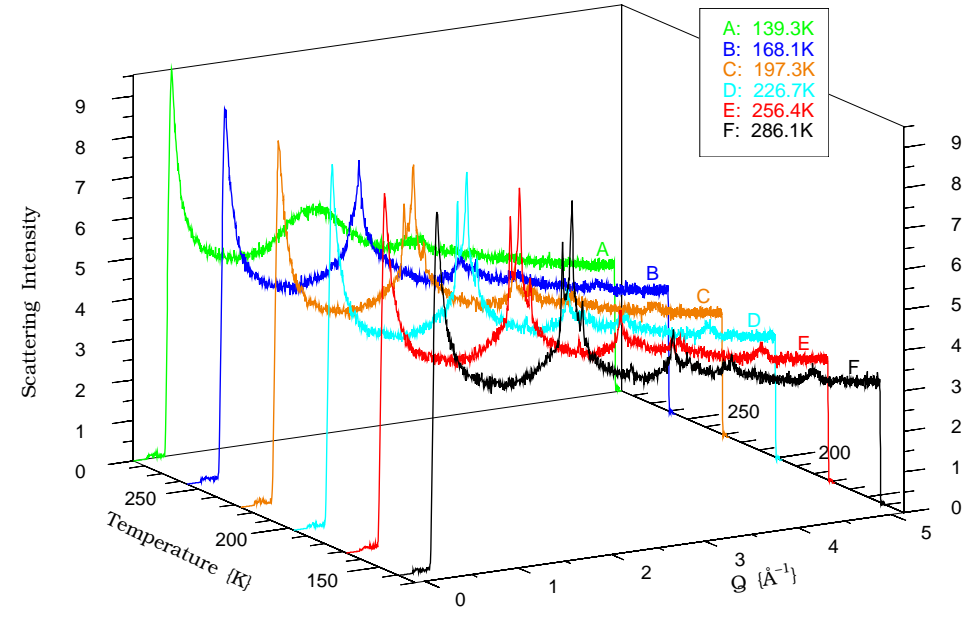

Figure 20: The diffraction pattern for water/ice in an under-filled sample of SBA-15 silica $(f=0.61)$ as a function of temperature during a warming ramp. Dore \& Webber, unpublished work [93].

ice-silica interface), the layers having a maximum thickness of about $3 \AA$. The maximum amount of hexagonal ice in the pore seems to be about $15 \%$ of the total pore volume.

Thus the small amount of hexagonal ice forming from the plastic ice, in the $\mathrm{f}=0.95$ study, see (Fig. 13), can be understood as the presence of a small fraction of the pores containing vapour, giving rise to plastic ice at the vapour surface, then converting to hexagonal ice. The slightly larger amount of plastic ice converting to hexagonal ice in the $f=1.31$ study (Fig. 12) must by this hypothesis be understood to indicate that this sample was not at equilibrium, and that a small percentage of pores were not fully filled when the experiment was performed. 


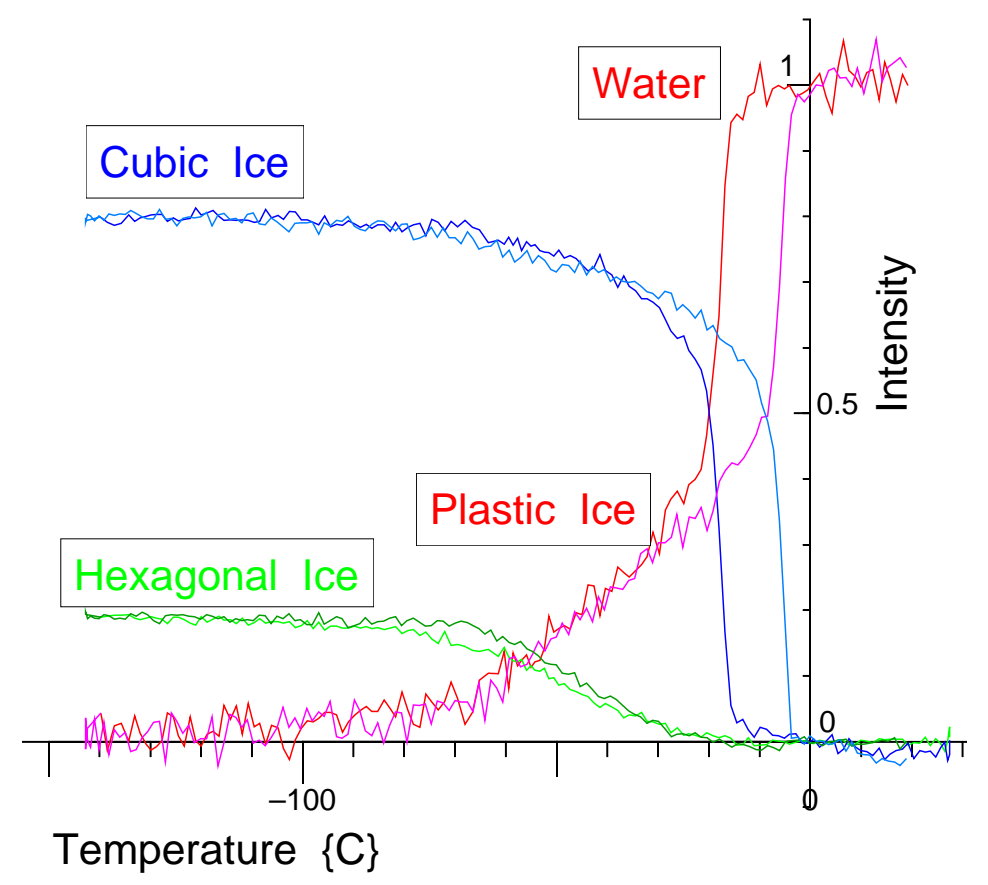

Figure 21: Normalised Neutron Diffraction Cryoporometry graphs of $\mathrm{D}_{2} \mathrm{O}$ water / ice fractions in SBA-15 templated silica: partially-filled, $f=0.61$. Graph reproduced with permission from the American Institute of Physics [92].

5.2.3. Structure, as determined be Neutron diffraction, using a peak analysis, with a partially-filled sample.

Curve fitting techniques were used to decompose the main ice peak triplet from the set of neutron scattering measurements as per Fig. 13, i.e. on a 'nearly filled' sample [88]; these indicate that, following the removal of any residual hexagonal ice scattering peaks, the remaining cubic peak be represented not as three peaks but as five, as shown in Fig. 22.

An analysis of the variation with temperature of the peak areas of the five fitted peaks plotted in Fig. 22 reveals that only one of the five (the 


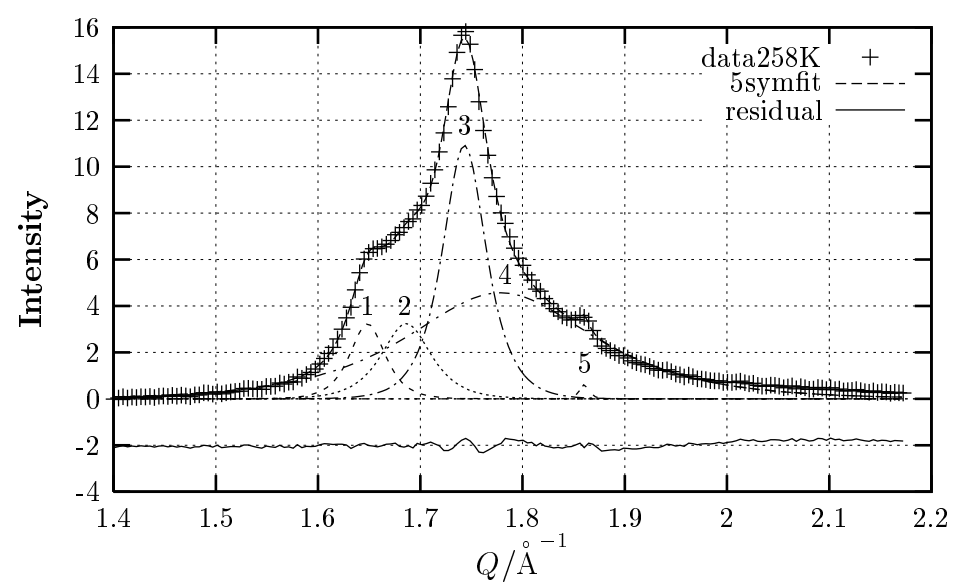

Figure 22: The fitted profile for the main peak using five symmetric peak functions at $258 \mathrm{~K}$, for the main neutron scattering peak for $\mathrm{D}_{2} \mathrm{O}$ in SBA-15. Graph reproduced with permission from Institute of Physics [88].

broad component peak 4), has a significant amplitude variation, as shown in Fig. 23, increasing at low temperature and reversibly decreasing as the temperature is again raised. It is worth noting that bulk amorphous ice may be prepared with a range of densities $[97,98,99,100,101]$ and Fig. 24 shows a set of diffraction data from low, intermediate and high density amorphous ice (lda, ida, hda) with $Q_{0}$ values ranging from 1.69 to $2.10 \AA^{-1}$ [102]. It is clear that the peak 4 in Fig. 22, with a $Q_{0}$ value of $1.768 \AA^{-1}$, is well matched in peak position by an intermediate density amorphous ice, but not in peak width.

Thus a tentative conclusion is that the disordered plastic ice component at a silica interface at least partially converts to an intermediate density amorphous ice, represented by fitted peak 4 of the cubic ice pattern in Fig. 22 , as the temperature is lowered, and then reversibly transforms back to the 


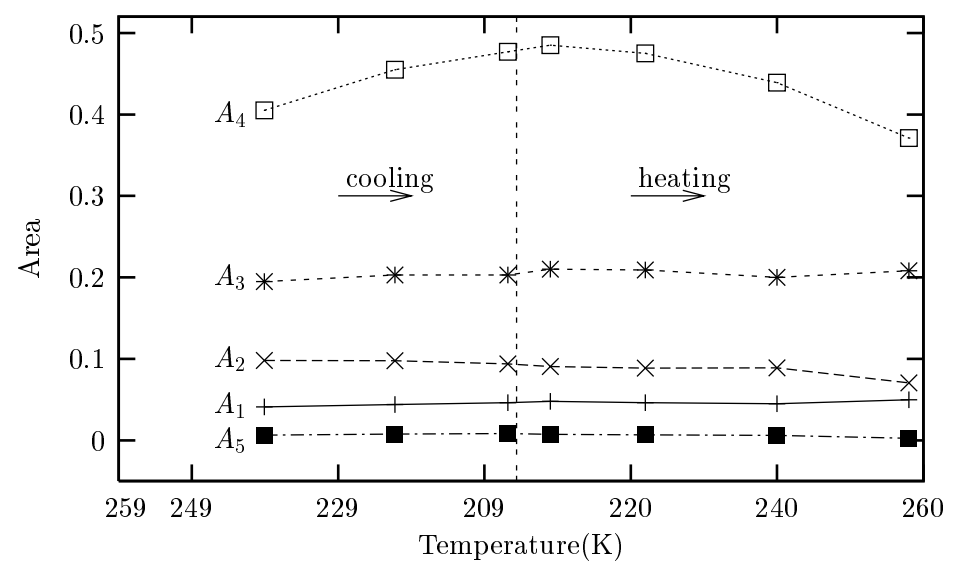

Figure 23: The variation of peak areas with temperature, $\mathrm{A}(\mathrm{T})$, for the five fitted peaks composing the main neutron scattering peak for $\mathrm{D}_{2} \mathrm{O}$ in SBA-15. Graph reproduced with permission from Institute of Physics [88].

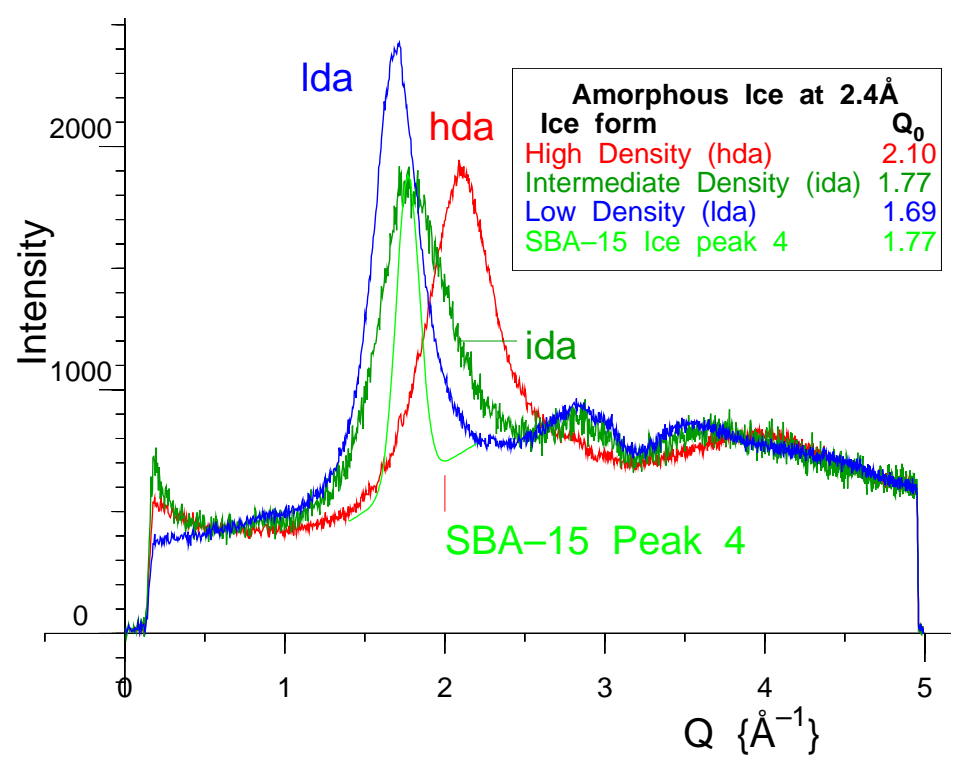

Figure 24: Diffraction patterns for bulk low, intermediate and high density amorphous ice (lda, ida, hda), as well as that of peak 4 in Fig. 22, for the ice in the SBA-15 sample. Graph reproduced with permission from Institute of Physics [88]. 
disordered form as the temperature is again raised. Further evidence for this conclusion is provided in the next section.

\subsubsection{Structure, as determined by Neutron diffraction, using a radial domain analysis.}

Neutron diffraction patterns measured for the scattering from $\mathrm{D}_{2} \mathrm{O}$ partially filling the pores in SPS-1 porous silica (nominal pore diameter $18 \AA$ ), is shown at a temperature where normally (in larger pores) cubic ice would be expected (Fig. 25). The scattering intensity at $150 \mathrm{~K}$ is also shown transformed to the radial domain (Fig. 26a), which shows a pattern that is extremely similar to a pattern measured previously for low-density amorphous ice [98] (Fig. 26b).

\section{Applications and Consequences.}

6.1. Relevance to NMR cryoporometry in short $T_{2}$ materials : meteorite example.

There is an interest in applying the technique of NMR cryoporometry to the study of rocks, clays and cements; in some of these environments there is low iron and paramagnetic content, and standard NMR-C techniques using water work well using $2 \tau$ echo sampling times of $2 \mathrm{~ms}$ or greater. In other environments metallic and paramagnetic components are present, $T_{2}$ and $T_{2}^{*}$ values are short, and there is a requirement to make measurements at $500 \mu \mathrm{s}$ or less. In these cases measurement using water as the probe liquid may be examining the brittle-plastic phase transition, rather than the brittle-liquid phase transition, thus leading to spuriously low pore-size 


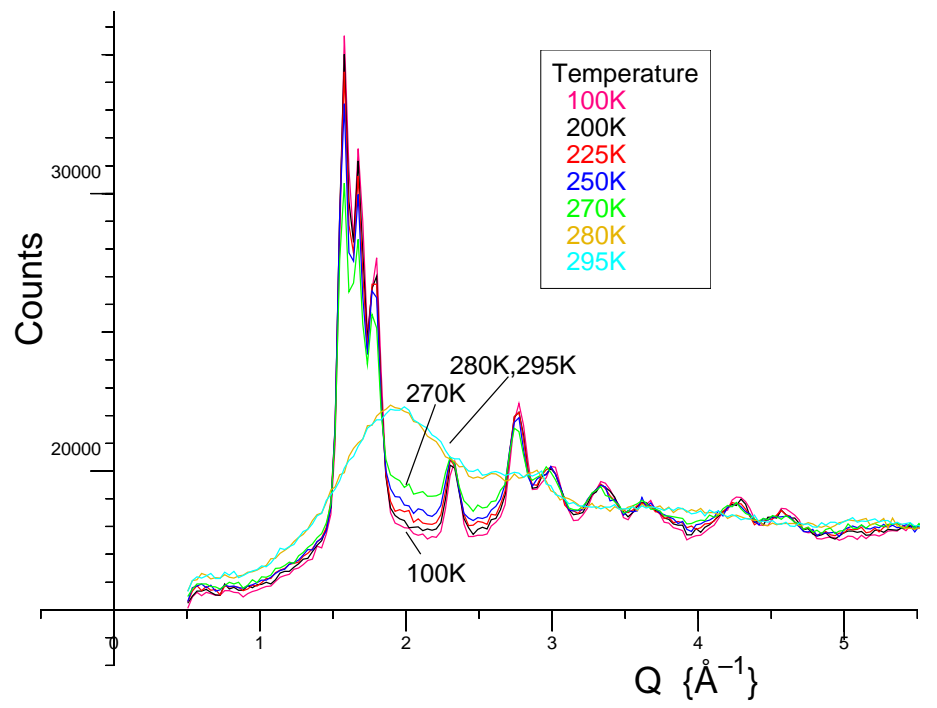

Figure 25: The variation of the diffraction pattern for $\mathrm{D}_{2} \mathrm{O}$ water in SPS-1 (partially filed) as a function of temperature during a warming ramp from $100 \mathrm{~K}$ to $295 \mathrm{~K}$, showing the main ice peaks exhibit negligible cubic ice, and also showing a conversion to disordered ice (Dore \& Webber, unpublished data). 


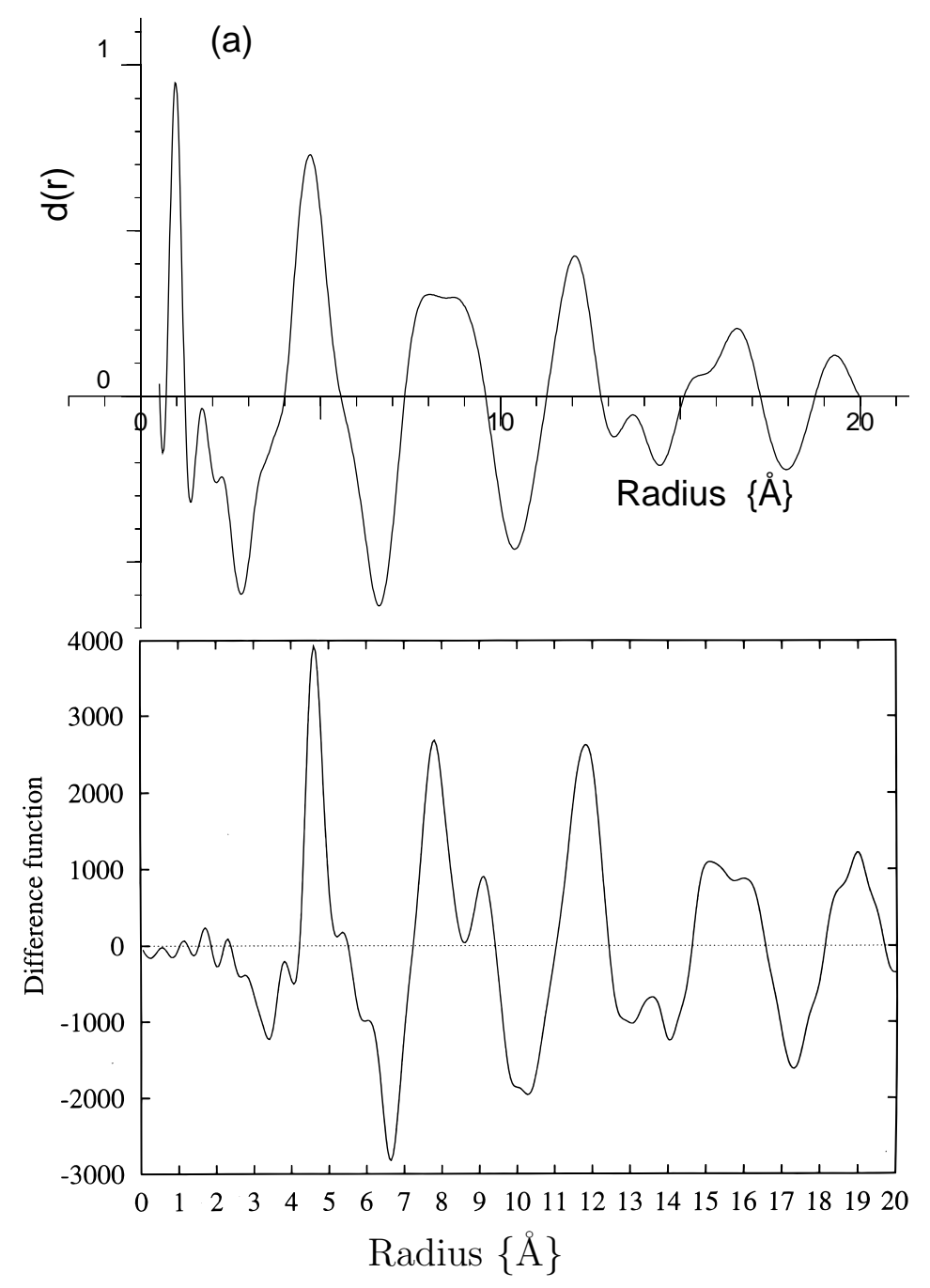

Figure 26: a) Radial distribution function $r \cdot G(r)=d(r)$ for $\mathrm{D}_{2} \mathrm{O}$ in SPS-1, at a temperature of $150 \mathrm{~K}$. b) $d(r)$ difference function for the transition from lda-ice to cubic ice at 140 K. Graphs reproduced with permission from Institute of Physics [87]. 
a)

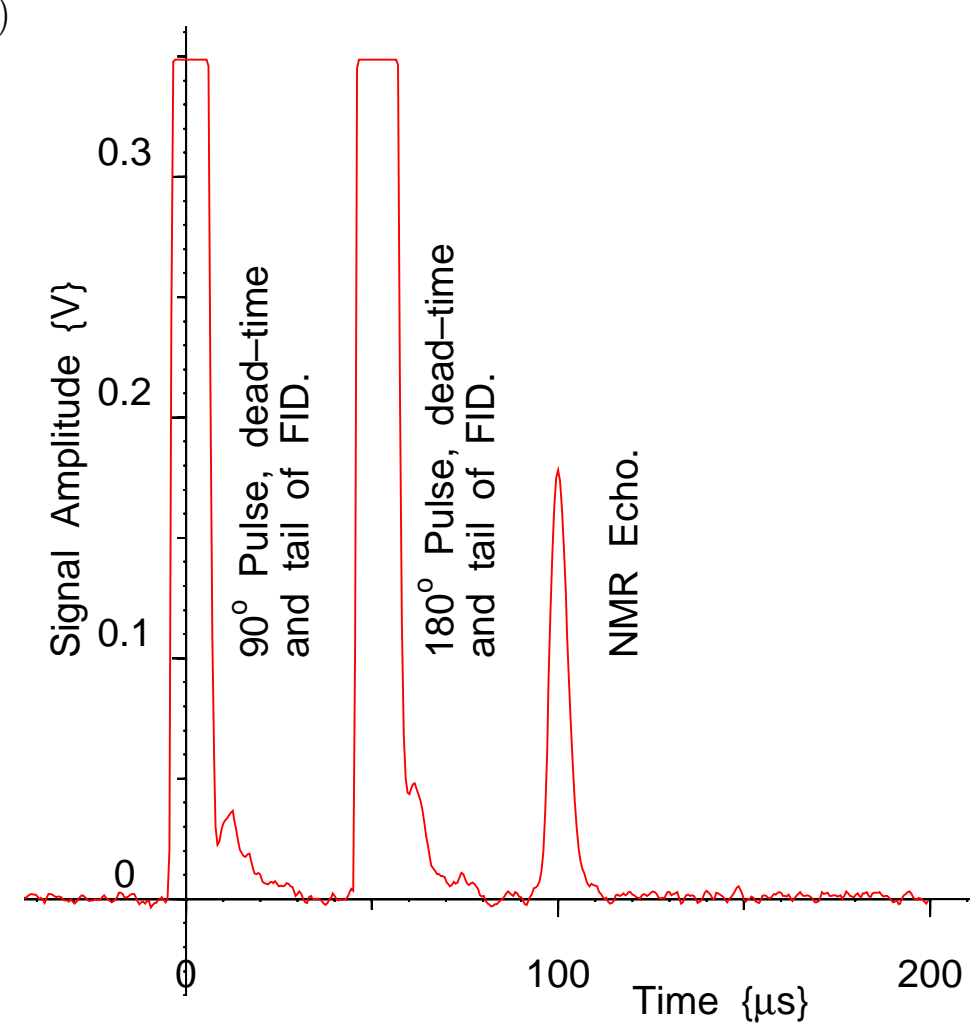

b)

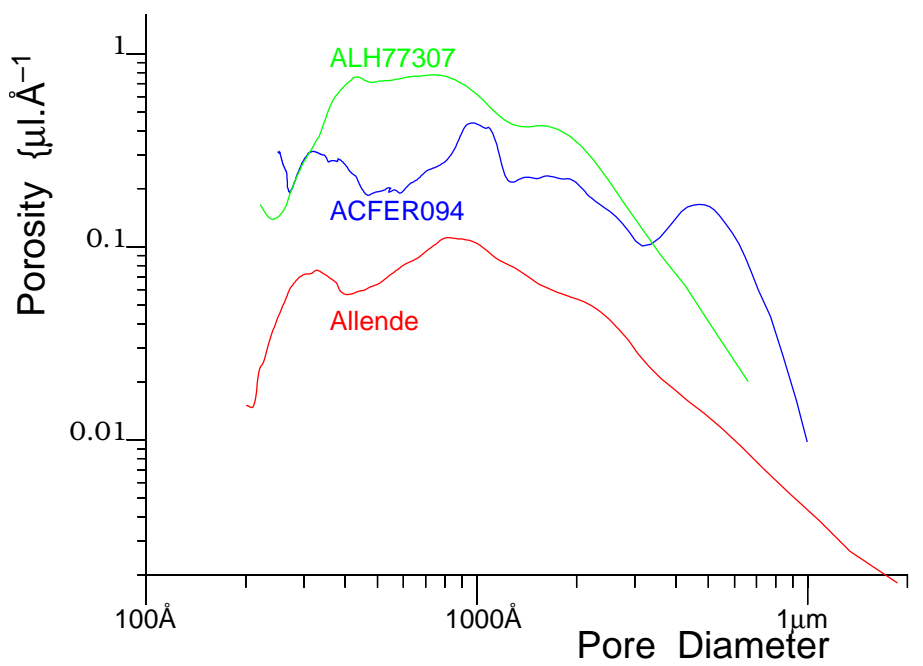

Figure 27: The pore-size distributions for these meteorite samples are measurable using an apolar liquid, but not water, due to the very short relaxation times being comparable with the $\mathrm{T}_{2}$ of plastic ice. a) NMR spin-echo traggfor an apolar liquid in meteorite ALH77307, showing extreme shortening of $T_{2}^{*}$ (HWHM decay time $=2.5 \mu \mathrm{s}$ in a static field $\mathrm{B}_{0}=0.5 \mathrm{~T}$ ) due to high magnetic gradients from the metallic content, with associated reduction in $T_{2}$. b) NMR cryoporometry pore-size distributions, measured at short $2 \tau$ times, using an apolar liquid without a significant plastic crystal component, for three meteorite samples : Allende, Acfer 094 and ALH77307 [92], Bland, Jackson, Coker, Cohen, Webber, Lee, Duffy, Chater, Ardakani, McPhail, McComb8 and Benedix : Unpublished results. 
measurements. Extreme examples of such short $T_{2}$ and short $T_{2}^{*}$ materials are provided by meteorites [103]. Fig. 27a shows the results from applying a spin-echo sequence for an apolar liquid in a meteoric sample, in a $0.5 \mathrm{~T}$ field, with the spin-echo trace revealing extreme broadening due to metallic component induced gradients. Fig. 27b shows that preliminary pore-size distributions can be successfully measured using NMR cryoporometry, using an a-polar liquid in meteoric samples [92], Bland, Jackson, Coker, Cohen, Webber, Lee, Duffy, Chater, Ardakani, McPhail, McComb8 and Benedix : Unpublished results. The latter paper considers the chemical evolution in meteorites over long periods and the evidence that this evolution is localised within the meteorite bodies rather than being a global average.

In addition, the enhanced dynamics that occur in ice at interfaces compared with the dynamical rates in bulk ice, as demonstrated in this discussion, may well be relevant to this long-time scale chemical evolution in meteorites and asteroidal bodies.

\subsection{Relevance to macroscopic snow-packs in the environment.}

A significant conclusion from both the NMR relaxation studies and the neutron diffraction cryoporometric studies is that plastic ice forms at icevapour interfaces, converting to hexagonal ice at low temperatures. It is well known from the study of the bulk mechanics of sea ice, snow-packs and glaciers, formed predominantly from hexagonal ice, that modeling must be done with either a viscous-plastic (VP) or an elastic-viscous-plastic (EVP) model [3]; this deduction that the ice may be in a state of enhanced rotational motion at each of the myriad of ice-vapour interfaces in compressed snowpacks, leading to a continual breaking and forming of the hydrogen bonds, 
may be relevant to these studies of ice and snow-packs in the environment [92].

\section{Acknowledgments}

Thanks are given to John Strange and John Dore for many productive discussions, and also to Lee Betteridge for the $\mathrm{N}_{2}$ gas adsorption calibration measurements. This study was partially supported by EPSRC grant $\mathrm{EP} / \mathrm{D} 052556 / 1$.

\section{References}

[1] J. D. Wright, N. A. J. M. Sommerdijk, Sol-Gel Materials: Chemistry and Applications, New Dover Edition, CRC Pr I Llc, 2000.

[2] E. Liu, J. C. Dore, Webber, D. Khushalani, S. Jähnert, G. H. Findenegg, T. Hansen, Neutron diffraction and NMR relaxation studies of structural variation and phase transformations for water/ice in SBA15 silica: I. the over-filled case, J. Phys.: Condens. Matter 18 (2006) 10009-10028. doi:10.1088/0953-8984/18/44/003.

[3] E. C. Hunke, J. K. Dukowicz, An elastic-viscous-plastic model for sea ice dynamics, J. Phys. Oceanogr. 27 (1997) 1849-1867.

[4] J. Gibbs, The scientific papers of J.Willward Gibbs, New Dover Edition, Vol. 1: Thermodynamics, Dover Publications, Inc., Constable and Co., New York, London, 1906 reprinted 1961.

[5] J. Gibbs, Collected Works, Longmans, Green and Co., New York, 1928. 
[6] J. Thomson, Theoretical considerations on the effect of pressure in lowering the freezing point of water, Trans. Roy. Soc. Edinburgh xvi (1849) 575-580.

[7] J. Thomson, On crystallization and liquefaction, as influenced by stresses tending to change form in the crystals, Proc. Roy. Soc. xi (1862) $473-481$.

[8] W. Thomson, On the equilibrium of vapour at a curved surface of liquid, Phil. Mag. 42 (1871) 448-452.

[9] J. Thomson, Application of dynamics to Physics and Chemistry, Macmillan \& Co, London, 1888.

[10] R. Defay, I. Prigogine, A. Bellemans, D. Everett, Surface tension and adsorption, English Edition, Longmans, Green \& Co Ltd., London, 1951, 1966.

[11] S. Gregg, K. Sing, Adsorption, Surface Area and Porosity, 2nd Edition, Academic Press, London, 1967.

[12] M. Coelingh, Optische onderzoekingen over het vloeistof-dampevenwicht in kapillaire stelsels, PhD, University of Utrecht. (1938).

[13] L. Cohan, Sorption hysteresis and the vapor pressure of concave surfaces, J. Am. Chem. Soc. 60 (1938) 433-435.

[14] C. Jackson, G. McKenna, The melting behavior of organic materials confined in porous solids, J. Chem. Phys. 93 (1990) 9002-9011. 
[15] T. Young, Phil. Trans. Roy. Soc. (London) 95 (1805) 65.

[16] T. Young, An essay on the cohesion of fluids, in: G. Peacock (Ed.), Miscellaneous Works of the late Thomas Young, Vol. 1, John Murray, Albemarle Street, London, 1855, Ch. XIX, pp. 418-453.

[17] J. L. Tell, H. J. Maris, Specific heats of hydrogen, deuterium, and neon in porous Vycor glass, Phys. Rev. B 28 (1983) 5122-5125. doi:10.1103/PhysRevB.28.5122.

[18] T. Martin, B. Lefevre, D. Brunel, A. Galarnea, F. D. Renz, F. Fajul, P. F. Gobi, J. F. Quinson, G. Vigier, Dissipative water intrusion in hydrophobic MCM-41 type materials, Chem. Commun. 1 (2002) 2425.

[19] J. Strange, M. Rahman, E. Smith, Characterisation of Porous Solids by NMR, Phys. Rev. Letts. 71 (1993) 3589-3591.

[20] J. B. W. Webber, J. C. Dore, J. H. Strange, R. Anderson, B. Tohidi, Plastic ice in confined geometry: The evidence from neutron diffraction and NMR relaxation., J. Phys.: Condens. Matter 19 (2007) 415117. doi:10.1088/0953-8984/19/41/415117.

[21] J. Webber, A generalisation of the thermoporisimetry Gibbs-Thomson equation for arbitrary pore geometry, Magn. Reson. Imaging 21 (2003) 428. doi:10.1016/S0730-725X(03)00172-3.

[22] O. Petrov, I. Furo, Curvature-dependent metastability of the solid phase and the freezing-melting hysteresis in pores, Phys. Rev. E 73 (2006) 011608 (7pp). doi:10.1103/PhysRevE.73.011608. 
[23] E. Barret, L. Joyner, P. Halenda, The determination of pore volume and area distributions in porous substances. i. computations from nitrogen isotherms, J. Am. Chem. Soc. 73 (1951) 373-380.

[24] J. Webber, The characterisation of porous media, PhD, Physics, University of Kent at Canterbury, UK.

(2000). URL http://www.kent.ac.uk/physical-sciences/publications/theses/jbww.html

[25] E. W. Hansen, R. Schmidt, M. Stocker, Pore structure characterization of porous silica by H-1 NMR using water, benzene, and cyclohexane as probe molecules, J. Phys. Chem-US 100 (1996) 11396-11401.

[26] S. Bahceli, A. Al-Kaisi, K. Krynicki, J. Strange, The effects of pore and particle geometry on NMR diffusion measurements in adsorbed liquids, in: F. Rodriguez-Reinoso, J. Rouquerol, K. Sing, K. Unger (Eds.), Characterisation of Porous Solids II, Vol. 62 of Studies in Surface Science and Catalysis, Elsevier, Amsterdam, 1991, pp. 293-300.

[27] S. Allen, M. Mallett, J. Strange, Morphology of porous media studied by nuclear magnetic resonance line shapes and spin-echo decays, J. Chem. Phys. 114 (2001) 3258-3264.

[28] N. Lisitza, Y. Song, Manipulation of the diffusion eigenmodes in porous media, Phys. Rev. B 65 (2002) 172406. doi:10.1103/PhysRevB.65.172406.

[29] R. C. Wilson, M. D. Huerlimann, Relationship between susceptibility induced field inhomogeneities, restricted diffusion, and re- 
laxation in sedimentary rocks, J. Magn. Reson. 183 (2006) 1-12. doi:10.1016/j.jmr.2006.07.006.

[30] J.-F. Kuntz, P. Palmas, V. Level, D. Canet, Restricted diffusion and exchange of water in porous media: Average structure determination and size distribution resolved from the effect of local field gradients on the proton NMR spectrum, J. Magn. Reson. 191 (2008) 239-247. doi:10.1016/j.jmr.2007.12.020.

[31] E. E. Sigmund, H. Cho, P. Chen, S. Byrnes, Y. Q. Song, X. E. Guo, T. R. Brown, Diffusion-based MR methods for bone structure and evolution, Magnet. Reson. Med. 59 (2008) 28-39. doi:10.1002/mrm.21281.

[32] K. E. Washburn, C. D. Eccles, P. T. Callaghan, The dependence on magnetic field strength of correlated internal gradient relaxation time distributions in heterogeneous materials, J. Magn. Reson. 194 (2008) 33-40. doi:10.1016/j.jmr.2008.05.025.

[33] K. Brownstein, C. Tarr, Spin-lattice relaxation in a system governed by diffusion, J. Magn. Resn. 26 (1977) 17-24.

[34] W. Halperin, S. Bhattacharja, F. d'Orazio, Relaxation and dynamic properties of water in partly filled porous media using NMR techniques, Mag. Res. Imag 9 (1991) 733-737.

[35] J. Strange, S. Allen, P. Stephenson, N. Matveeva, Phase equilibria of absorbed liquids and the structure of porous media, Magn. Reson. Imaging. 14 (1996) 963-965. doi:10.1016/S0730-725X(96)00196-8. 
[36] S. Stapf, R. Kimmich, R.-O. Seitter, Field-cycling NMR relaxometry of liquids confined in porous glass: Evidence for Levy-walks, Magnetic Resonance Imaging 14 (1996) 841-846.

[37] S. Allen, P. Stephenson, J. Strange, Internal surfaces of porous media studied by nuclear magnetic resonance cryoporometry, J. Chem. Phys. 108 (1998) 8195-8198. doi:10.1063/1.476175.

[38] H. Booth, J. Strange, Microdynamics and phase equilibria in organic nanocrystals, Magn. Reson. Imaging. 16 (1998) 501-504. doi:10.1016/S0730-725X(98)00066-6.

[39] H. Booth, J. Strange, Organic nanocrystals: an NMR study of cyclohexane in porous silica, Mol. Phys. 93 (1998) 263-269. doi:10.1080/002689798169267.

[40] P. Levitz, J.-P. Korb, D. Petit, Slow dynamics of embedded fluid in mesoscopic confining systems as probed by NMR relaxometry, Eur. Phys. J. E. 12 (2003) 29-33.

[41] J. Strange, J. Mitchell, J. Webber, Pore surface exploration by nmr, Magn. Reson. Imaging 21 (2003) 221-226. doi:10.1016/S0730$725 \mathrm{X}(03) 00128-0$.

[42] S. Alnaimi, J. Mitchell, J. Strange, J. Webber, Binary liquid mixtures in porous solids, J. Chem. Phys. 120 (2004) 2075-2077. doi:10.1063/1.1643730.

[43] P. Sebastiao, D. Sousa, A. Ribeiro, M. Vilfan, G. Lahajnar, J. Seliger, S. Zumer, Field-cycling NMR relaxometry of a liquid crystal above 
T-Ni in mesoscopic confinement, Phys. Rev. E 72 (2005) 061702. doi:10.1103/PhysRevE.72.061702.

[44] H. Jaffel, J.-P. Korb, J.-P. Ndobo-Epoy, J.-P. Guicquero, V. Morin, Multi-scale approach continuously relating the microstructure and the macroscopic mechanical properties of plaster pastes during their settings, J. Phys. Chem. 110 (2006) 18401-18407. doi:10.1021/jp062832a.

[45] J.-P. Korb, Surface diffusion of liquids in disordered nanopores and materials: A field cycling relaxometry approach, in: Conner, WC and Fraissard, J (Ed.), Fluid Transport in Nanoporous Materials, Vol. 219 of Nato Science Series, Series II: Mathematics, Physics And Chemistry, 2006, pp. 415-437, Conference of the NATO-Advanced-StudyInstitute on Fluid Transport in Nanoporous Materials, La Colle sur Loup, France, Jun 16-28, 2003.

[46] P. J. McDonald, J. Mitchell, M. Mulheron, P. S. Aptaker, J.-P. Korb, L. Monteilhet, Two-dimensional correlation relaxometry studies of cement pastes performed using a new one-sided NMR magnet, Cement Concrete Res. 37 (2007) 303-309. doi:10.1016/j.cemconres.2006.01.013.

[47] W. Schoenfelder, H.-R. Glaeser, I. Mitreiter, F. Stallmach, Twodimensional NMR relaxometry study of pore space characteristics of carbonate rocks from a Permian aquifer, J. Appl. Geophys. 65 (2008) 21-29. doi:10.1016/j.jappgeo.2008.03.005.

[48] K. Overloop, L. van Gerven, Freezing Phenomena in Adsorbed Wa- 
ter as Studied by NMR, J. Magn. Reson. 101 (1993) 179-187. doi:10.1006/jmra.1993.1028.

[49] S. Alnaimi, J. Strange, E. Smith, The characterisation of porous solids by nmr, Magn. Reson. Imaging 12 (1994) 257-259.

[50] E. Hansen, M. Stocker, R. Schmidt, Low-Temperature Phase Transition of Water Confined in Mesopores Probed by NMR. Influence on Pore Size Distribution., J. Phys. Chem. US 100 (1996) 2195-2200.

[51] J. Webber, J. Strange, J. Dore, An evaluation of NMR Cryoporometry, Density Measurement and Neutron Scattering methods of pore characterisation, Mag. Res. Imag. 19 (2001) 395-399. doi:10.1016/S0730725X(01)00255-7.

[52] J. Mitchell, S. Stark, J. Strange, Probing surface interactions by combining NMR cryoporometry and NMR relaxometry, J. Phys. D. Appl. Phys. 38 (2005) 1950-1958. doi:10.1088/0022-3727/38/12/015.

[53] J.-D. Jeon, S.-Y. Kwak, Ionic cluster size distributions of swollen nafion/sulfated beta-cyclodextrin membranes characterized by nuclear magnetic resonance cryoporometry, J. Phys. Chem. 111 (2007) 94379443. doi:10.1021/jp070980a.

[54] A. Khokhlov, R. Valiullin, J. Kaerger, F. Steinbach, A. Feldhoff, Freezing and melting transitions of liquids in mesopores with ink-bottle geometry, New J. Phys. 9 (2007) 272. doi:10.1088/1367-2630/9/8/272.

[55] D. Vargas-Florencia, I. Furo, R. W. Corkery, Pore morphology and 
interconnectivity in a mesoporous/macroporous polyhedral silica foam material, Langmuir 24 4827-4832. doi:10.1021/la702318y.

[56] J. Mitchell, J. B. W. Webber, J. Strange, Nuclear Magnetic Resonance Cryoporometry, Phys. Rep. 461 (2008) 1-36. doi:10.1016/j.physrep.2008.02.001.

[57] O. V. Petrov, I. Furo, NMR cryoporometry: Principles, applications and potential, Prog. Nucl. Mag. Res. Sp. 54 (2009) 97-122. doi:10.1016/j.pnmrs.2008.06.001.

[58] A. Mitzithras, F. Coveney, J. Strange, NMR-studies of the diffusion of cyclohexane in porous silica, J. Mol. Liq. 54 (1992) 273-281.

[59] S. Codd, P. Callaghan, Spin echo analysis of restricted diffusion under generalized gradient waveforms: Planar, cylindrical, and spherical pores with wall relaxivity, J. Magn. Reson. 137 (1999) 358-372.

[60] F. Stallmach, J. Kärger, The potentials of pulsed field gradient NMR for investigation of porous media, Adsorption-Journal of The International Adsorption Society 5 (1999) 117-133.

[61] H. Paoli, A. Methivier, H. Jobic, C. Krause, H. Pfeifer, F. Stallmach, J. Kärger, Comparative QENS and PFG NMR diffusion studies of water in zeolite NaCaA, Micropor. Mesopor. Mat. 55 (2002) 147-158.

[62] R. Kimmich, Strange kinetics, porous media, and NMR, Chem. Phys. 284 (2002) 253-285. doi:10.1016/S0301-0104(02)00552-9. 
[63] P. Callaghan, S. Godefroy, B. Ryland, Diffusion-relaxation correlation in simple pore structures, J. Magn. Reson. 162 (2003) 320-327.

[64] R. Valiullin, I. Furo, V. Skirda, P. Kortunov, NMR magnetization transfer as a tool for characterization of nanoporous materials, Magn. Reson. Imaging 21 (2003) 299-303, 6th International Conference on the Recent Advances in Magnetic Resonance Applications to Porous Media, Ulm, Germany, Sep 08-12, 2002. doi:10.1016/S0730725X(03)00158-9.

[65] J. Petkovic, H. Huinink, L. Pel, K. Kopinga, Diffusion in porous building materials with high internal magnetic field gradients, J. Magn. Reson. 167 (2004) 97-106. doi:10.1016/j.jmr.2003.11.007.

[66] M. Flaum, G. Hirasaki, C. Flaum, C. Straley, Measuring pore connectivity by pulsed field gradient diffusion editing with hydrocarbon gases, Magn. Reson. Imaging 23 (2005) 337-339, 7th International Conference on Magnetic Resonance in Porous Media (MRPM7), Palaiseau, France, Jul 04-08, 2004. doi:10.1016/j.mri.2005.01.002.

[67] G. Farrher, I. Ardelean, R. Kimmich, Probing four orders of magnitude of the diffusion time in porous silica glass with unconventional NMR techniques, J. Magn. Reson. 182 (2006) 215-220. doi:10.1016/j.jmr.2006.06.032.

[68] H. Jobic, W. Schmidt, C. Krause, J. Kärger, PFG NMR and QENS diffusion study of n-alkane homologues in MFI- 
type zeolites, Micropor. Mesopor. Mat. 90 (2006) 299-306. doi:10.1016/j.micromeso.2005.10.020.

[69] I. Ardelean, G. Farrhera, R. Kimmich, Effective diffusion in partially filled nanoscopic and microscopic pores, J. Optoelectron. Adv. 9 (2007) 655-660, International Conference on Advanced Spectroscopies on Biomedical and Nanostructured Systems, Cluj Napoca, Romania, Sep 03-06, 2006.

[70] J.-F. Kuntz, P. Palmas, D. Canet, Diffusive diffraction measurements in porous media: Effect of structural disorder and internal magnetic field gradients, J. Magn. Reson. 188 (2007) 322-329. doi:10.1016/j.jmr.2007.08.009.

[71] G. Farrher, I. Ardelean, R. Kimmich, Time-dependent molecular diffusion in partially filled porous glasses with heterogeneous structure, Appl. Magn. Reson. 34 (2008) 85-99, 22nd Conference on Radio and Microwave Spectroscopy (RAMIS 2007), Bedlewo, Poland, APR 22-25, 2007. doi:10.1007/s00723-008-0096-6.

[72] O. Geier, R. Q. Snurr, F. Stallmach, J. Kärger, Boundary effects of molecular diffusion in nanoporous materials: A pulsed field gradient nuclear magnetic resonance study, J. Chem. Phys. 120 (2004) 367-373. doi:10.1063/1.1629276.

[73] D. Majolino, C. Corsaro, V. Crupi, V. Venuti, U. Wanderlingh, Water diffusion in nanoporous glass: An NMR study at different hydration levels, J. Phys. Chem. B 112 (2008) 3927-3930. doi:10.1021/jp711433d. 
[74] J. C. Gore, A. W. Anderson, M. D. Does, D. F. Gochberg, J. M. Joers, R. P. Kennan, E. C. Parsons, M. Schachter, The relationship of problems in biomedical MRI to the study of porous media, Magn. Reson. Imaging 19 (2001) 295-300. doi:10.1016/S0730-725X(01)002399 .

[75] J. Mitchell, J. Strange, An NMR investigation of naphthalene nanostructures, Mol. Phys. 102 (2004) 1997-2005. doi:10.1080/00268970412331292795.

[76] L. Monteilhet, J. P. Korb, J. Mitchell, P. J. McDonald, Observation of exchange of micropore water in cement pastes by two-dimensional T-2-T-2 nuclear magnetic resonance relaxometry, Phys. Rev. E 74. doi:10.1103/PhysRevE.74.061404.

[77] A. Schreiber, I. Ketelsen, G. Findenegg, Melting and freezing of water in ordered mesoporous silica materials, Phys. Chem. Chem. Phys. 3 (2001) 1185-1195.

[78] J. B. W. Webber, J. C. Dore, Neutron Diffraction Cryoporometry a measurement technique for studying mesoporous materials and the phases of contained liquids and their crystalline forms., Nucl. Instrum. Meth. A. 586 (2008) 356-366. doi:10.1016/j.nima.2007.12.004.

[79] S. Jaehnert, F. V. Chavez, G. E. Schaumann, A. Schreiber, M. Schoenhoff, G. H. Findenegg, Melting and freezing of water in cylindrical silica nanopores, Phys. Chem. Chem. Phys. 10 (2008) 6039-6051. doi:10.1039/b809438c. 
[80] J. Dore, B. Webber, J. Strange, H. Farman, M. Descamps, L. Carpentier, Phase transformations for cyclohexane in mesoporous silicas, Physica A 333 (2004) 10-16. doi:10.1016/j.physa.2003.09.043.

[81] O. Petrov, I. Furo, Curvature-dependent metastability of the solid phase and the freezing-melting hysteresis in pores, Phys. Rev. E 73 (2006) 011608.

[82] J. B. W. Webber, R. Anderson, J. H. Strange, B. Tohidi, Clathrate formation and dissociation in vapour/water/ice/hydrate systems in SBA15, Sol-Gel and CPG porous media, as probed by NMR relaxation, novel protocol NMR Cryoporometry, Neutron Scattering and ab-initio QM-MD simulation, in: 8th International Meeting on Recent Advances in MR Applications to Porous Media, Bologna, Italy, 10th to 14th September, 2006., 2006.

[83] J. B. W. Webber, R. Anderson, J. H. Strange, B. Tohidi, Clathrate formation and dissociation in vapour/water/ice/hydrate systems in SBA-15, Sol-Gel and CPG porous media, as probed by NMR relaxation, novel protocol NMR Cryoporometry, Neutron Scattering and ab-initio quantum-mechanical molecular dynamics simulation., Magn. Reson. Imaging 25 (2007) 533-536. doi:10.1016/j.mri.2006.11.022.

[84] G. Mason, Determination of the pore-size distributions and pore-space interconnectivity of Vycor porous glass from adsorption-desorption hysteresis capillary condensation isotherms., Proceedings of the Royal Society of London A 415 (1988) 453-486. 
[85] H. Christenson, Confinement effects on freezing and melting, J. Phys.: Condens. Matter 13 (2001) R95-R133.

[86] V. M. Gun'ko, V. V. Turov, V. I. Zarko, E. V. Goncharuk, I. I. Gerashchenko, A. A. Turova, I. F. Mironyuk, R. Leboda, J. Skubiszewska-Zieba, W. Janusz, Comparative characterization of polymethylsiloxane hydrogel and silylated fumed silica and silica gel, J. Colloid Interf. Sci. 308 (2007) 142-156. doi:10.1016/j.jcis.2006.12.053.

[87] J. Seyed-Yazdi, Farman, J. Dore, J. B. W. Webber, G. Findenegg, T. Hansen, Structural characterization of water and ice in mesoporous SBA-15 silicas: II. The 'almost-filled' case for $86 \AA$ pore diameter, J. Phys.: Condens. Matter 20 (2008) 205107. doi:10.1088/0953$8984 / 20 / 20 / 205107$.

[88] J. Seyed-Yazdi, H. Farman, J. Dore, J. B. W. Webber, G. Findenegg, Structural characterization of water and ice in mesoporous SBA-15 silicas: III. The triplet profile for $86 \AA$ pore diameter, J. Phys.: Condens. Matter 20 (2008) 205108. doi:10.1088/0953-8984/20/20/205108.

[89] S. Brunauer, P. Emmett, E. Teller, Adsorption of gases in multimolecular layers, J. Am. Chem. Soc. 60 (1938) 309-319.

[90] M. Brun, A. Lallemand, J. Quinson, C. Eyraud, A new method for the simultaneous determination of the size and the shape of pores: The Thermoporometry, Thermochimica Acta 21 (1977) 59-88.

[91] B. Webber, J. Dore, Structural and dynamic studies of water in mesoporous silicas using neutron scattering and nuclear magnetic resonance, 
J. Phys.: Condens. Matter 16 (2004) S5449-S5470. doi:10.1088/0953$8984 / 16 / 45 / 009$.

[92] J. B. W. Webber, J. H. Strange, P. A. Bland, R. Anderson, B. Tohidi, Dynamics at Surfaces: Probing the Dynamics of Polar and A-Polar Liquids at Silica and Vapour Surfaces, American Institute of Physics (AIP) Conference Proceedings Series 1081 (2008) 51-54. doi:10.1063/1.3058545.

[93] J. B. W. Webber, J. H. Strange, P. A. Bland, R. Anderson, B. Tohidi, Dynamics at Surfaces: Probing the Dynamics of Polar and A-Polar Liquids at Silica and Vapour Surfaces, in: 9th International Meeting on Recent Advances in MR Applications to Porous Media, 13th to 17th July, 2008, 2008.

[94] J. Chezeau, J. Strange, Diffusion in Molecular-crystals, Phys. Rep. 53 (1979) 1-92.

[95] A. Sah, H. L. Castricum, A. Bliek, D. H. A. Blank, J. E. ten Elshof, Hydrophobic modification of gamma-alumina membranes with organochlorosilanes, Journal Of Membrane Science 243 (2004) 125-132.

[96] J. Zanotti, M. Bellissent-Funel, S. Chen, Experimental evidence of a liquid-liquid transition in interfacial water, Europhys. Lett. 71 (2005) 91-97.

[97] M. R. Chowdhury, J. C. Dore, J. T. Wenzel, The structural characteristics of amorphous D2O ice by neutron-diffraction, J. Non-Cryst. Solids 53 (1982) 247-265. 
[98] D. Blakey, Structural studies of vapour-deposited amorphous-ice and argon/amorphous-ice systems by neutron diffraction, $\mathrm{PhD}$ thesis, University of Kent at Canterbury, UK. (1994).

[99] T. Loerting, C. Salzmann, I. Kohl, E. Mayer, A. Hallbrucker, A second distinct structural "state" of high-density amorphous ice at 77K and 1 bar, Phys. Chem. Chem. Phys. 3 (2001) 5355-5357.

[100] J. L. Finney, A. Hallbrucker, I. Kohl, A. K. Soper, D. T. Bowron, Structures of high and low density amorphous ice by neutron diffraction, Phys. Rev. Lett. 88 (2002) 225503.

[101] J. L. Finney, D. T. Bowron, A. K. Soper, T. Loerting, E. Mayer, A. Hallbrucker, Structure of a new dense amorphous ice, Phys. Rev. Lett. 89 (2002) 205503.

[102] M. M. Koza, T. Hansen, R. P. May, H. Schober, Link between the diversity, heterogeneity and kinetic properties of amorphous ice structures, J. Non-Cryst. Solids 352 (2006) 4988-4993.

[103] P. A. Bland, O. Alard, G. K. Benedix, A. T. Kearsley, O. N. Menzies, L. Watt, N. W. Rogers, Volatile fractionation in the early solar system and chondrule/matrix complementarity., Proc. Natl Acad. Sci. USA 102 (2005) 13755-13760. 